\title{
Luna's fundamental lemma for diagonalizable groups
}

\author{
Dan Abramovich and Michael Temkin
}

\begin{abstract}
We study relatively affine actions of a diagonalizable group $G$ on locally noetherian schemes. In particular, we generalize Luna's fundamental lemma when applied to a diagonalizable group: we obtain criteria for a $G$-equivariant morphism $f: X^{\prime} \rightarrow X$ to be strongly equivariant, namely the base change of the morphism $f / / G$ of quotient schemes, and establish descent criteria for $f / / G$ to be an open embedding, étale, smooth, regular, syntomic, or lci.
\end{abstract}

\section{Introduction}

\subsection{Luna's fundamental lemma}

In its original formulation, Luna's fundamental lemma [Lun73, Lemme fondamental] addresses the following classical question: we are given an étale equivariant morphism $f: X^{\prime} \rightarrow X$ between two normal complex varieties with the action of a reductive algebraic group $G$. We assume that there are quotients $Y=X / / G$ and $Y^{\prime}=X^{\prime} / / G$ with a resulting quotient morphism $f / / G: Y^{\prime} \rightarrow Y$. Under what conditions is $f$ strongly étale, namely $X^{\prime}=Y^{\prime} \times_{Y} X$ and $f / / G$ is étale? In other words, when is the following diagram cartesian and the bottom row étale as well?

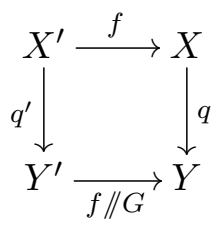

\subsection{Generalized context}

In this paper, we restrict attention to diagonalizable groups, relax all the other conditions, and deduce further properties of the quotient:

(1) Our schemes are only required to be locally noetherian, with no assumption on being normal or even reduced.

(2) The action of $G$ on $X$ and $X^{\prime}$ is assumed to be relatively affine in the sense of Section 5.1.

(3) We provide necessary and sufficient conditions for an arbitrary equivariant morphism $f$ to be strongly equivariant in the sense that $f$ is the base change of $f / / G$.

Received 24 September 2016, accepted in final form 1 January 2017.

2010 Mathematics Subject Classification 14L30.

Keywords: diagonalizable groups, Luna's fundamental lemma.

This journal is (C) Foundation Compositio Mathematica 2018. This article is distributed with Open Access under the terms of the Creative Commons Attribution Non-Commercial License, which permits non-commercial reuse, distribution, and reproduction in any medium, provided that the original work is properly cited. For commercial re-use, please contact the Foundation Compositio Mathematica.

This research is supported by BSF grant 2010255. 


\section{Abramovich and M. Temkin}

(4) We establish the descent of various properties of $f$, ensuring that $f / / G$ is regular, smooth, étale, or syntomic (without any finite type assumption) when $f$ has the corresponding property.

Condition (2) guarantees the existence of good quotients $Y$ and $Y^{\prime}$; see Section 5.1. It also guarantees that over any point $y \in Y$, the fiber $X_{y}$ contains a unique closed orbit, called here a special orbit (Section 5.1.8).

Already in the complex case, most equivariant morphisms are not strongly equivariant: one must require something about how $f$ treats stabilizers and special orbits. Accordingly, we say that $f: X^{\prime} \rightarrow X$ is fiberwise inert (Section 5.5.3) if it sends special orbits to special orbits and if for each point $x \in X$, the stabilizer $G_{x}$ acts trivially on the fiber $X^{\prime} \times_{X} x$.

\subsection{The main result}

The main result of this article is the following variant of Luna's fundamental lemma.

Theorem 1.3.1 (see Theorem 5.6.4). Let $X$ and $X^{\prime}$ be locally noetherian schemes provided with relatively affine actions of a diagonalizable group $G$, and let $f: X^{\prime} \rightarrow X$ be a $G$-equivariant morphism. Then:

(i) The morphism $f$ is strongly equivariant if and only if $f$ is fiberwise inert and any special orbit in $X^{\prime}$ contains a point $x^{\prime}$ such that the action of $G_{x^{\prime}}$ on $H_{1}\left(\mathbb{L}_{X^{\prime} / X} \otimes^{\mathrm{L}} k\left(x^{\prime}\right)\right)$ is trivial.

(ii) Let $P$ be one of the following properties: (a) regular, (b) smooth, (c) étale, (d) an open embedding. Then the following conditions are equivalent: (1) $f$ is inert and satisfies $P,(2) f$ is strongly equivariant and satisfies $P,(3) f$ strongly satisfies $P$.

(iii) The morphism $f$ is strongly syntomic if and only if it is syntomic and strongly equivariant. Moreover, if $f$ is strongly equivariant, then the following claims hold: (a) if $f$ is lci, then $f / / G$ is lci, (b) if $f / / G$ is lci and Tor-independent with the morphism $X \rightarrow X / / G$, then $f$ is lci.

Part (iii) refers to Avramov's definition of local complete intersection (lci) morphisms and the resulting notion of syntomic morphisms described in Section 4.7.9.

Theorem 1.3.1 is the first ingredient in our forthcoming work [AT16] on the weak factorization of birational maps, generalizing the main theorems of [AKMW02] and [Wło03] to the appropriate generality of qe ("quasi-excellent") schemes, and further proving factorization results in other geometric categories of interest. While [AT16] only requires actions of $\mathbb{G}_{m}$, we find it both convenient and fruitful to work with an arbitrary diagonalizable group. A second ingredient for [AT16], called torification, is developed in the appropriate generality in [AT17], which builds on this paper.

In these applications of Theorem 1.3.1, it is crucial that $X$ and $X^{\prime}$ are allowed to be arbitrary locally noetherian schemes, not necessarily varieties over a field. Part (iii) of the theorem is required for results of [AT17] in mixed characteristics: we apply it to charts of logarithmically regular schemes, which are lci but in general not flat.

Beyond these applications, we found it striking that a sharp condition for strong equivariance such as statement (i), for morphisms $f$ which are not even lci, was at all possible.

\subsection{Comparison with the literature}

Luna's original work applied to normal complex varieties with reductive group actions, and it was extended to algebraically closed field of arbitrary characteristics by Bardsley and Richard- 


\section{LUNA'S FUNDAMENTAL LEMMA FOR DIAGONALIZABLE GROUPS}

son; see [BR85]. In both works, the only condition one has to impose on an étale equivariant morphism $f$ is pointwise inertia preservation.

Recently, Alper generalized Luna's fundamental lemma to the context of good moduli spaces of Artin stacks; see [Alp10, Theorem 6.10]. In this situation, the morphism from a stack to its moduli space is an analogue of the morphism $X \rightarrow X / / G$, and Alper studied the classical question of describing strongly étale morphisms. In this generality, an additional condition has to be imposed on $f$, and the weak saturation condition introduced in [Alp10, Section 2.2] is analogous to our condition on special orbits. Comparing to our results, we see that he proves only an extension of the classical Luna's fundamental lemma concerning étale morphisms, but the context is much more general in two aspects: stacks as opposed to schemes acted on by groups, and the class of groups involved (or stabilizers of stacks).

\subsection{Further directions}

It would be interesting, perhaps in future work, to further extend our strong variant of Luna's fundamental lemma to other tame actions, that is, actions having linearly reductive (or even reductive) stabilizers and affine orbits. Moreover, one may hope to extend this to tame groupoids and their quotients, that one may call tame stacks (if the stabilizers are of dimension zero, then those are the tame stacks of [AOV08]). For example, a simple descent argument shows that all results of this paper hold for groups of multiplicative type; see Section 5.7.

\subsection{Auxiliary results}

On the way to proving Theorem 1.3.1, we study related notions of regularity, formal smoothness, and group actions. Some results which may be of independent interest and will be used in [AT17] are recorded here. The reader interested only in Theorem 1.3.1 may wish to skip directly to Section 2.

1.6.1 The splitting of formally smooth morphisms. We review in Section 2 the notions of regularity and formal smoothness. In particular, Section 2.2.10 introduces the notion of a Cohen ring $C(k) \rightarrow k$ of a field $k$; further, by [SP17, tag/032A], any formally smooth $g: k \rightarrow D$ with $D$ a complete noetherian $k$-algebra lifts to a formally smooth morphism we denote by $C(g): C(k) \rightarrow$ $C(D)$, where $C(D)$ is a complete noetherian local ring such that $D=C(D) \otimes_{C(k)} k$. This applies in characteristic zero by taking $C(k)=k$ and $C(D)=D$. The following result describes an arbitrary formally smooth homomorphism in terms of such $C(g)$.

TheOrem 1.6.2 (see Theorem 2.2.11). Let $f: A \rightarrow B$ be a local homomorphism of complete noetherian local rings with closed fiber $\bar{f}: k=A / m_{A} \rightarrow \bar{B}=B / m_{A} B$.

(i) Assume that $\bar{f}$ is formally smooth. Then there exist homomorphisms $i: C(k) \rightarrow A$ and $j: C(\bar{B}) \rightarrow B$ making the following diagram commutative:

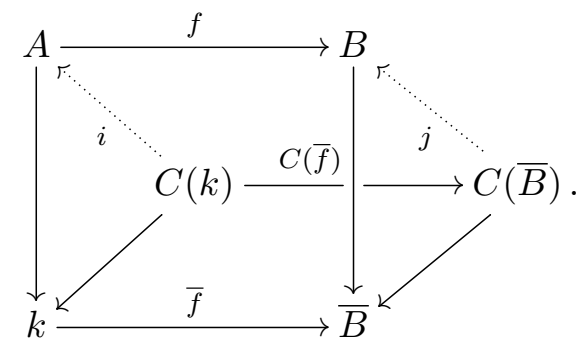




\section{Abramovich and M. Temkin}

(ii) Assume that $f$ is formally smooth. Then for any choice of $i$ and $j$, the homomorphism $A \widehat{\otimes}_{C(k)} C(\bar{B}) \rightarrow B$ is an isomorphism. In particular, $f$ is (non-canonically) isomorphic to the formal base change of a Cohen lift $C(\bar{f})$ of its closed fiber $\bar{f}$.

1.6.3 L-local rings. After reviewing groups, actions, and diagonalizable groups in Section 3, we study diagonalizable group actions on affine schemes in Section 4. Consider a finitely generated abelian group $L$ and its Cartier dual $G=\mathbf{D}_{L}$. An action of $G$ on an affine scheme $X=\operatorname{Spec} A$ is an $L$-grading on $A$. An important class of $L$-graded rings that we study is the class of $L$-local rings, namely rings $A$ possessing a single maximal $L$-homogeneous ideal $m_{A}$; see Section 4.4 . The residue ring $A / m_{A}$ has no non-trivial homogeneous ideals, making it a "graded field." Several standard results on local rings generalize to this setting, including the following.

Proposition 1.6.4 (Graded Nakayama's lemma, see Proposition 4.4.10 and Corollary 4.4.11). Let $(A, m)$ be an $L$-local ring with residue graded field $A / m_{A}$, and let $M$ be a finitely generated $L$-graded A-module. Then:

(i) We have $m M=M$ if and only if $M=0$.

(ii) A homogeneous homomorphism of L-graded $A$-modules $\phi: N \rightarrow M$ is surjective if and only if $\phi \otimes_{A}\left(A / m_{A}\right)$ is surjective.

(iii) Homogeneous elements $m_{1}, \ldots, m_{l}$ generate $M$ if and only if their images generate $M / m M$.

(iv) The minimal cardinality of a set of homogeneous generators of $M$ equals the rank of the free $\left(A / m_{A}\right)$-module $M / m M$.

Proposition 1.6.5 (Characterization of equivariant Cartier divisors, see Proposition 4.4.13). Assume that $(A, m)$ is an $L$-local integral domain and $D \subset \operatorname{Spec}(A)$ is an equivariant, finitely presented closed subscheme. Let $x$ be an arbitrary point of $V(m)$, and let $X_{x}=\operatorname{Spec}\left(\mathcal{O}_{X, x}\right)$ be the localization at $x$. Then the following conditions are equivalent:

(i) We have $D=V(f)$ for a homogeneous element $f \in A$.

(ii) The subscheme $D$ is a Cartier divisor in $X$.

(iii) The localization $D_{x}=D \times_{X} X_{x}$ is a Cartier divisor in $X_{x}$.

To characterize the situation discussed above, we say that a relatively affine action of $G$ on a scheme $X$ is local if $X$ is quasi-compact and contains a single closed orbit; see Section 5.1.9.

Proposition 1.6.6 (Characterization of local actions, see Lemma 5.1.10). Assume that we have a relatively affine action of $G=\mathbf{D}_{L}$ on a scheme $X$. Then the following conditions are equivalent:

(i) The action is local.

(ii) The scheme $X$ is affine, say $X=\operatorname{Spec}(A)$, and the $L$-graded ring $A$ is $L$-local.

(iii) The quotient $Y=X / / G$ is local.

1.6.7 Completions. By an $L$-complete local ring, we mean a complete local ring $(A, m)$ provided with a formal L-grading $A=\prod_{n \in L} A_{n}$ such that $A_{n} \subset m$ for each $n \neq 0$. Here is a key result about completions which is used in the paper to prove the formal version of Luna's fundamental lemma; see Theorem 4.7.8.

Proposition 1.6.8 (Completions of $L$-local rings, see Proposition 4.5.6). Assume that $L$ is a finitely generated abelian group and $(A, m)$ is a noetherian strictly $L$-local ring. Set $m_{0}=$ 


\section{LUNA'S FUNDAMENTAL LEMMA FOR DIAGONALIZABLE GROUPS}

$m \cap A_{0}$ and for each $n \in L$, let $\widehat{A}_{n}$ denote the $m_{0}$-adic completion of the $A_{0}$-module $A_{n}$. Then, the $m$-adic completion of $A$ is isomorphic to $\prod_{n \in L} \widehat{A}_{n}$; in particular, it is an L-complete local ring.

1.6.9 Quotients and strong equivariance. Using the affine theory as a starting point, a global theory of relatively affine actions is developed in Section 5, leading to the main theorem. We highlight here the following basic results.

Theorem 1.6.10 (Properties preserved by quotients, see Theorem 5.2.2). Assume that a diagonalizable group $G=\mathbf{D}_{L}$ acts trivially on a scheme $S$ and an $S$-scheme $X$ is provided with a relatively affine action of $G$.

(i) Assume that $X$ satisfies one of the following properties: (a) reduced, (b) integral, (c) normal with finitely many connected components, (d) locally of finite type over $S$, (e) of finite type over $S$, (f) quasi-compact over $S$, (g) locally noetherian, (h) noetherian. Then $X / / G$ satisfies the same property.

(ii) If $X$ is locally noetherian, then the quotient morphism $X \rightarrow X / / G$ is of finite type.

Proposition 1.6.11 (Preservation of strong equivariance, see Lemma 5.3.2). Let $G=\mathbf{D}_{L}$ be a diagonalizable group.

(i) The composition of strongly $G$-equivariant morphisms is strongly $G$-equivariant.

(ii) If $Y \rightarrow X$ is a strongly $G$-equivariant morphism and $g: Z \rightarrow Y$ is a $G$-equivariant morphism such that the composition is strongly $G$-equivariant, then $g$ is strongly $G$-equivariant.

(iii) If $Y \rightarrow X$ is strongly $G$-equivariant and $Z \rightarrow X$ is $G$-equivariant, then the base change $Y \times_{X} Z \rightarrow Z$ is strongly $G$-equivariant.

(iv) If $f: Y \rightarrow X$ is strongly equivariant, then the diagonal $\Delta_{f}: Y \rightarrow Y \times_{X} Y$ is strongly equivariant and $\Delta_{f} / / G$ is the diagonal of $f / / G$.

\section{Regularity and formal smoothness}

We recall in this section basic facts about formal smoothness in the local case, which is the only case we will use later. The cited results are due to Grothendieck, but we will cite [SP17] in addition to [EGA]. In addition, we will prove in Theorems 2.2.9 and 2.2.11 a splitting result for local formally smooth homomorphisms, which seems to be new, although it is close in spirit to what was known.

\subsection{Definitions}

2.1.1 Regular morphisms. Regular morphisms are a generalization of smooth morphisms in situations of morphisms which are not necessarily of finite type. Following [EGA, $\mathrm{IV}_{2}, 6.8 .1$, a morphism of schemes $f: Y \rightarrow X$ is said to be regular if

- the morphism $f$ is flat and

- all geometric fibers of $f: X \rightarrow Y$ are regular.

2.1.2 Formal smoothness of local homomorphisms. Let $f:\left(A, m_{A}\right) \rightarrow\left(B, m_{B}\right)$ be a local homomorphism of local rings. By saying that $f$ is formally smooth, we mean that it is formally smooth with respect to the $m_{A}$-adic and $m_{B}$-adic topologies. This means that for any $\operatorname{ring} C$ 


\section{Abramovich and M. Temkin}

with a square zero ideal $I$ and compatible homomorphisms $A \rightarrow C$ and $B \rightarrow C / I$ that vanish on large powers of $m_{A}$ and $m_{B}$, respectively,

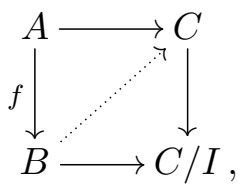

there exists a lifting $B \rightarrow C$ making the diagram commutative. Using a slightly non-standard terminology, we say that a morphism of schemes $f: Y \rightarrow X$ is formally smooth at $y \in Y$ if the local homomorphism $\mathcal{O}_{X, f(y)} \rightarrow \mathcal{O}_{Y, y}$ is formally smooth.

Remark 2.1.3. Unlike regularity, formal smoothness is not a local property. Consider an example of a discrete valuation ring (DVR) which is not excellent in characteristic $p$, namely a DVR $R$ such that $\widehat{K}=\operatorname{Frac}(\widehat{R})$ is not separable over $K=\operatorname{Frac}(R)$. For example, set $\widehat{R}=k[[x]]$, take an element $y \in k[[x]]$ which is transcendental over $k(x)$, and set $R=\widehat{R} \cap k\left(x, y^{p}\right)$. Then $\widehat{R}$ is, indeed, the completion of $R$, and the homomorphism $R \rightarrow \widehat{R}$ is formally smooth but its generic fiber $K \rightarrow \widehat{K}$ is not.

2.1.4 Formally factorizable homomorphisms. Following [FR93], we say that a homomorphism of noetherian local rings $\phi: A \rightarrow B$ is formally factorizable if its completion can be factored into a composition of a formally smooth homomorphism of noetherian complete local rings $\widehat{A} \rightarrow D$ and a surjective homomorphism $D \rightarrow \widehat{B}$. Any such factorization $\widehat{A} \rightarrow D \rightarrow \widehat{B}$ will be called a formally smooth factorization of $\widehat{\phi}$. It is proved in [FR93, Theorem 4] that $\phi$ is formally factorizable if and only if the extension of the residue fields $l / k$ has a finite-dimensional imperfection module $\Upsilon_{l / k}=H_{1}\left(\mathbb{L}_{l / k}\right.$ ) (see [EGA, $0_{\text {IV }}, 20.6 .1$ and 21.4.8]).

Remark 2.1.5. Note that $\operatorname{dim}_{l} \Upsilon_{l / k}=\infty$ can happen only when $k$ has infinite $p$-rank (that is, $\left[k: k^{p}\right]=\infty$ ) and the extension $l / k$ is not finitely generated. In particular, formally non-factorizable homomorphisms are pretty exotic and almost never show up in applications.

\subsection{Properties of formal smoothness}

2.2.1 Criteria. Here are the main criteria for the formal smoothness of local homomorphisms of noetherian rings.

Theorem 2.2.2. For a local homomorphism $f:(A, m) \rightarrow(B, n)$ of noetherian local rings, the following conditions are equivalent:

(i) The morphism $f$ is formally smooth.

(ii) The completion $\widehat{f}: \widehat{A} \rightarrow \widehat{B}$ is formally smooth.

(ii)' The completion $\widehat{f}: \widehat{A} \rightarrow \widehat{B}$ is a regular homomorphism.

(iii) The morphism $f$ is flat and its closed fiber $\bar{f}: k=A / m \rightarrow \bar{B}=B / m B$ is formally smooth.

(iii)' The morphism $f$ is flat and its closed fiber $\bar{f}: k=A / m \rightarrow \bar{B}=B / m B$ is a regular homomorphism (that is, $\bar{B}$ is geometrically regular over $k$ ).

Proof. The equivalence of statements (i) and (ii) is almost obvious; see [SP17, tag/07ED]. See [SP17, tag/07NQ] for the equivalence of statements (ii), (iii), and (iii)', and see [SP17, tag/07PM] for the equivalence of statements (ii) and (ii)'.

Corollary 2.2.3. A morphism of noetherian schemes $f: Y \rightarrow X$ is regular if and only if it is formally smooth at all points of $Y$. 


\section{LUNA'S FUNDAMENTAL LEMMA FOR DIAGONALIZABLE GROUPS}

Proof. First, assume that $f$ is formally smooth at all points of $Y$, so statement (i) above is satisfied locally at every point. By the theorem, statement (iii)' is satisfied locally at every point, so $f$ is flat and the geometric fibers are locally regular, hence regular. Being flat with regular geometric fibers, the morphism $f$ is regular.

The other direction is similar: assume that $f$ is regular, so it satisfies statement (iii)' of the theorem at every point. By the theorem, statement (i) holds at every point, namely $f$ is formally smooth at every point.

As another corollary, we obtain that formal smoothness behaves nicely for qe (that is, "quasiexcellent") schemes (for example, see [ILO14, Definition I.2.10]). In particular, it becomes a local property (this is due to André, see [And74]).

Corollary 2.2.4. Assume that $\phi: A \rightarrow B$ is a local homomorphism of noetherian rings and $A$ is a qe ring. Then $\phi$ is formally smooth if and only if it is regular. In particular, if $f: Y \rightarrow X$ is a morphism of noetherian schemes with $X$ a qe scheme and if $f$ is formally smooth at all closed points of $Y$, then $f$ is regular.

Proof. Assume that $\phi$ is formally smooth. By Theorem 2.2.2, the completion $\widehat{\phi}: \widehat{A} \rightarrow \widehat{B}$ is regular. Since $A$ is quasi-excellent, the composition $A \rightarrow \widehat{A} \rightarrow \widehat{B}$ is regular. Since $B$ is noetherian, the completion homomorphism $B \rightarrow \widehat{B}$ is flat and surjective on spectra. By [SP17, tag/07NT], the homomorphism $\phi$ is regular.

The other direction follows from Theorem 2.2.2 or Corollary 2.2.3.

2.2.5 An adic lifting property. It is shown in [SP17, tag/07NJ] that a formally smooth $f$ satisfies a strong lifting property with respect to continuous homomorphisms to adic rings. We will need the following particular case.

Lemma 2.2.6. Let $f: A \rightarrow B$ be a formally smooth local homomorphism, let $C$ be a complete noetherian local ring, and let $I \subset C$ be any ideal. Then any pair of compatible homomorphisms of topological rings $A \rightarrow C$ and $B \rightarrow C / I$ admits a lifting $B \rightarrow C$.

Proof. Since $C$ is noetherian, $I$ is a closed ideal; see [Mat89, Theorem 8.14]. Also, $I$ is contained in the maximal ideal of $C$, which is an ideal of definition. Therefore the claim follows from [SP17, $\operatorname{tag} / 07 \mathrm{NJ}]$.

Corollary 2.2.7. Let $A$ be a local ring with residue field $k$, let $B$ and $C$ be complete noetherian local $A$-algebras, and assume that $B$ is formally smooth over $A$. Then an $A$-homomorphism $g: C \rightarrow B$ is an isomorphism if and only if its closed fiber $\bar{g}=g \otimes_{A} k$ is an isomorphism.

Proof. Only the inverse implication needs a proof. We first claim that if the closed fiber $\bar{g}$ is surjective, then $g$ is surjective. Since $B$ and $C$ are complete with respect to their maximal ideals, they are also complete with respect to $m_{A} B$ and $m_{A} C$, respectively. As $\bar{g}$ is surjective, the $(n-1)$ st infinitesimal fibers $C / m_{A}^{n} C \rightarrow B / m_{A}^{n} B$ are surjective by Nakayama's lemma for nilpotent ideals, and we obtain that $g$ is surjective too.

Setting $I=\operatorname{Ker}(g)$ and using Lemma 2.2.6, we obtain that there exists a homomorphism $h: B \rightarrow C$ making the following diagram commutative:

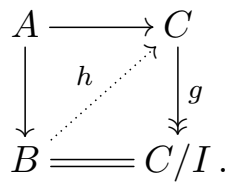




\section{Abramovich and M. Temkin}

In other words, we have found a section $h$ of $g$. Note that the closed fiber of $h$ is surjective (in fact, $\left.\bar{h}=\bar{g}^{-1}\right)$. The first statement proven above with $g$ replaced by $h$ gives that $h$ is surjective.

2.2.8 The splitting of formally smooth homomorphisms. Now we are ready to prove the main result of Section 2 in the equal characteristic case: any formally smooth homomorphism between complete noetherian local rings is a base change of its closed fiber. For convenience of the exposition, we will deal with the mixed-characteristic case separately.

Recall that if $A$ is a complete noetherian local ring with residue field $k$ and if $A$ contains a field, then $A$ contains $k$ as a coefficient field $i: k \hookrightarrow A$ by Cohen's structure theorem [SP17, $\operatorname{tag} / 032 \mathrm{~A}]$.

Theorem 2.2.9. Let $f: A \rightarrow B$ be a local homomorphism of complete noetherian local rings with closed fiber $\bar{f}: k=A / m_{A} \rightarrow \bar{B}=B / m_{A} B$. Assume that $A$ contains a field.

(i) Assume that $\bar{f}$ is formally smooth. Then there exist a coefficient field $i: k \rightarrow A$ and a section $j: \bar{B} \hookrightarrow B$ of the surjection $B \rightarrow \bar{B}$ which extends the composition $f \circ i: k \hookrightarrow A \rightarrow B$.

(ii) Assume that $f$ is formally smooth. Then for any choice of such $i$ and $j$, the homomorphism $A \widehat{\otimes}_{k} \bar{B} \rightarrow B$ is an isomorphism. In particular, $f$ is (non-canonically) isomorphic to the formal base change of its closed fiber $\bar{f}$ with respect to $i$.

Proof. To establish the existence of $j$, we apply Lemma 2.2.6 to the following diagram:

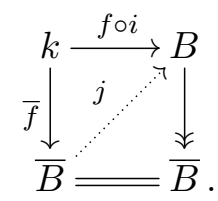

Once $j$ is fixed, we obtain a homomorphism $g: A \widehat{\otimes}_{k} \bar{B} \rightarrow B$ of complete noetherian local $A$ algebras whose closed fiber $\bar{g}: k \otimes_{k} \bar{B} \stackrel{\sim}{\rightarrow} \bar{B}$ is an isomorphism. Hence $g$ is an isomorphism by Corollary 2.2.7.

2.2.10 The mixed-characteristic case. Recall that given a field $k$ with $\operatorname{char}(k)=p>0$, a Cohen ring $C(k)$ is a complete DVR with residue field $k$ and maximal ideal $(p)$. Since $C(k)$ is formally smooth over $\mathbb{Z}_{p}$ (see Theorem 2.2.2), for a complete local ring $A$ with $A / m_{A}=k$, the homomorphism $C(k) \rightarrow k$ lifts to $f: C(k) \rightarrow A$. In fact, this argument is used in the proof of Cohen's structure theorem [SP17, tag/032A]: in the mixed-characteristic case, $C(k) \hookrightarrow A$ is a ring of coefficients of $A$, and in the equal-characteristic case, $\operatorname{Im}(f)=k$ is a field of coefficients of $A$.

Assume that $k$ is of characteristic $p>0$. By [SP17, tag/07NR], any formally smooth homomorphism $g: k \rightarrow D$ with $D$ a complete noetherian $k$-algebra admits a formally smooth lifting $f: C(k) \rightarrow E$, with $E$ a suitable complete noetherian ring, in the sense that $g=f \otimes_{C(k)} k$. Moreover, if $f^{\prime}: C(k) \rightarrow E^{\prime}$ is another such lifting, then, by the formal smoothness of $f$, the homomorphism $E \rightarrow D$ lifts to a homomorphism $E \rightarrow E^{\prime}$, which is necessarily an isomorphism. For this reason, we will use the notation $C(D)=E$ and $C(g)=f$.

In order to unify the notation, if $R$ is a ring containing $\mathbb{Q}$, we set $C(R)=R$, and for any homomorphism $f: R \rightarrow S$, we set $C(f)=f$. Here is the analogue of Theorem 2.2.9.

Theorem 2.2.11. Let $f: A \rightarrow B$ be a local homomorphism of complete noetherian local rings with closed fiber $\bar{f}: k=A / m_{A} \rightarrow \bar{B}=B / m_{A} B$. 


\section{LUNA'S FUNDAMENTAL LEMMA FOR DIAGONALIZABLE GROUPS}

(i) Assume that $\bar{f}$ is formally smooth. Then there exist homomorphisms $i: C(k) \rightarrow A$ and $j: C(\bar{B}) \rightarrow B$ making the following diagram commutative:

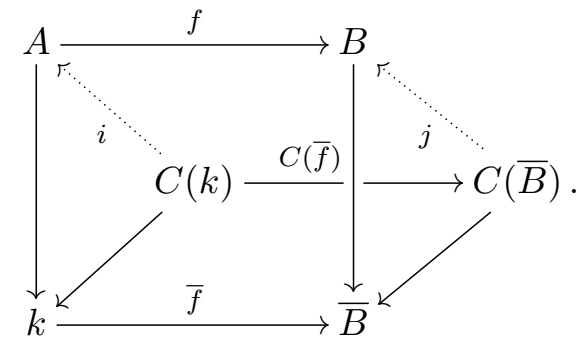

(ii) Assume that $f$ is formally smooth. Then for any choice of $i$ and $j$, the homomorphism $A \widehat{\otimes}_{C(k)} C(\bar{B}) \rightarrow B$ is an isomorphism. In particular, $f$ is (non-canonically) isomorphic to the formal base change of a Cohen lift $C(\bar{f})$ of its closed fiber $\bar{f}$.

Proof. As we saw at the beginning of Section 2.2.10, the homomorphism $C(k) \rightarrow k$ lifts to $i: C(k) \rightarrow A$. In particular, $B$ becomes a $C(k)$-algebra. Since $C(\bar{f})$ is formally smooth by Theorem 2.2.2, the $C(k)$-homomorphism $C(\bar{B}) \rightarrow \bar{B}$ lifts to a $C(k)$-homomorphism $j: C(\bar{B}) \rightarrow B$ by Lemma 2.2.6.

Given $i$ and $j$, we obtain a homomorphism $g: A \widehat{\otimes}_{C(k)} C(\bar{B}) \rightarrow B$ of complete noetherian local $A$-algebras whose closed fiber $\bar{g}: k \otimes_{k} \bar{B} \stackrel{\sim}{\rightarrow} \bar{B}$ is an isomorphism. Hence $g$ is an isomorphism by Corollary 2.2.7.

Remark 2.2.12. In a sense, Theorem 2.2.9 reduces the classification of formally smooth homomorphisms $f: A \rightarrow B$ of complete local rings to the case when the source is a field. By Theorem 2.2.2, $g: k \rightarrow \Lambda$ is formally smooth if and only if $\Lambda$ is geometrically regular over $k$. Perhaps this is the "best" characterization of formally smooth $k$-algebras one can give in general. On the other hand, if we further assume that $K=\Lambda / m_{\Lambda}$ is separable over $k$ (for example, if $k$ is perfect), then a better characterization is possible: $g$ is formally smooth if and only if $\Lambda$ is of the form $K \llbracket t_{1}, \ldots, t_{n} \rrbracket$. Indeed, since $k \rightarrow K$ is formally smooth, we can extend $g$ to a field of coefficients $K \hookrightarrow \Lambda$. Then we choose $t_{1}, \ldots, t_{n}$ to be any family of regular parameters. Applying Theorem 2.2.11, we obtain as a consequence that if $f$ is formally smooth and the extension of the residue fields $K / k$ is separable, then there is an isomorphism of $A$-algebras $A \widehat{\otimes}_{C(k)} C(K) \llbracket t_{1}, \ldots, t_{n} \rrbracket \stackrel{\sim}{\rightarrow} B$.

\section{Generalities on group scheme actions}

In this section, we fix some basic terminology, including group schemes, orbits, stabilizers, etc.

\subsection{General groups}

3.1.1 Group schemes and actions. A $\mathbb{Z}$-flat group scheme (respectively, flat $S$-group scheme) $G$ will be simply referred to as a group (respectively, $S$-group). An action of a group $G$ on a scheme $X$ is given by an action morphism $\mu: G \times X \rightarrow X$ satisfying the usual compatibilities: associativity and the triviality of the unit action. Similarly, an action of an $S$-group $G$ on an $S$-scheme $X$ is given by a morphism $\mu: G \times_{S} X \rightarrow X$ satisfying the analogous requirements. If $X$ is an $S$-scheme and $G$ is a group, then an action of $G$ on $X$ is called an $S$-action if $\mu$ is an $S$-morphism. Giving such an action is equivalent to providing $X$ with an action of the $S$-group $G_{S}=G \times S$.

Remark 3.1.2. The projection and the action morphisms give rise to a groupoid $G \times X \rightrightarrows X$ for 


\section{Abramovich and M. Temkin}

the flat, locally of finite presentation (fppf) topology, see [SP17, tag/0234], which often shows up in constructions related to the action.

3.1.3 Stabilizers. The stabilizer or inertia group of an action of $G$ on $X$ is the $X$-group scheme

$$
I_{X}=\operatorname{Eq}\left(G_{X} \rightrightarrows X\right)=G_{X} \underset{X \times X}{\times} X
$$

where the component maps of $G_{X} \rightarrow X \times X$ are the action and projection maps and $X \rightarrow X \times X$ on the right is the diagonal. This is a subgroup of the $X$-group $G_{X}$, which is often not flat. For any point $x \in X$, we define its stabilizer as the fiber $G_{x}=I_{X} \times_{X} \operatorname{Spec}(k(x))$.

3.1.4 Orbits. Working with varieties, one usually considers only "classical" orbits of group actions, which can be characterized as orbits of closed points or locally closed orbits. When $G$ acts on a more general scheme $X$, it is more natural to take into account orbits of all points. A set-theoretic orbit of $x \in X$ is the image of the map $G \times \operatorname{Spec}(k(x)) \rightarrow X$. This definition ignores the non-reduced structure which becomes essential when $G$ is non-reduced. For example, free and non-free actions of $\mu_{p}$ are distinguished by the nilpotent structure of the orbits.

In order to define scheme-theoretic orbits, one should use the scheme-theoretic image; see [Har77, II, Exercise 3.11(d)], [SP17, tag/01R6]. Let $\bar{O}_{x}$ be the scheme-theoretic image of $G \times$ $\operatorname{Spec}(k(x)) \rightarrow X$, and let $O_{x}$ be obtained from $\bar{O}_{x}$ by removing all proper closed subsets of the form $\bar{O}_{y}$. We provide $\bar{O}_{x}$ with the structure sheaf $\left.\mathcal{O}_{\bar{O}_{x}}\right|_{O_{x}}$ and call it the $G$-orbit of $x$. We do not know general criteria for $O_{x}$ to be a scheme, but the orbits we will use below are in fact schemes. Note that in this case, $O_{x}$ is a limit of open subschemes of $\bar{O}_{x}$.

\subsection{Diagonalizable groups}

Starting from this point, we consider only diagonalizable groups $G$. Probably, many results can be extended to the case of arbitrary linearly reductive or even reductive groups, but we do not pursue that direction.

3.2.1 The definition. By a diagonalizable group, we mean a finite-type diagonalizable group $G$ over $\mathbb{Z}$; see [SGA3-2, VIII.1.1]. In other words, $G=\mathbf{D}_{L}=\operatorname{Spec}(\mathbb{Z}[L])$ for a finitely generated abelian group $L$. Note that for any subgroup $L^{\prime} \subseteq L$ with the quotient $L^{\prime \prime}=L / L^{\prime}$, we have a natural embedding $\mathbf{D}_{L^{\prime \prime}} \hookrightarrow \mathbf{D}_{L}$ and $\mathbf{D}_{L} / \mathbf{D}_{L^{\prime \prime}} \stackrel{\sim}{\rightarrow} \mathbf{D}_{L^{\prime}}$. Moreover, this construction exhausts all subgroups and quotient groups of $\mathbf{D}_{L}$.

3.2.2 The action of a diagonalizable group. Any element $d \in L$ induces a character $\chi_{d}: G \rightarrow$ $\mathbb{G}_{m}=\mathbf{D}_{\mathbb{Z}}$, and this construction identifies $L$ with the group of all characters of $G$. An action of a diagonalizable group $G=\mathbf{D}_{L}$ on a scheme $X$ can also be described in the dual language by giving a comultiplication homomorphism of $\mathcal{O}_{X}$-algebras $\mu^{\#}: \mathcal{O}_{X} \rightarrow \mathcal{O}_{X}[L]$.

3.2.3 The affine case. If $X=\operatorname{Spec}(A)$ is affine, then the action is described by the homomorphism $\mu^{\#}: A \rightarrow A[L]$, and it is easy to see that such a homomorphism $\mu^{\#}$ is, indeed, a comultiplication if and only if it corresponds to an $L$-grading $A=\oplus_{n \in L} A_{n}$ on $A$, see [SGA3-1, I.4.7.3]; if $a=\sum a_{n}$ with $a_{n} \in A_{n}$, then $\mu^{\#}(a)=\sum a_{n} n$. From here on, we will identify $G$-actions on $X=\operatorname{Spec}(A)$ with the corresponding $L$-gradings of $A$. 


\section{LUNA'S FUNDAMENTAL LEMMA FOR DIAGONALIZABLE GROUPS}

\section{4. $L$-graded rings}

In this section, we study how a diagonalizable group $G=\mathbf{D}_{L}$ acts on affine schemes $X=\operatorname{Spec}(A)$. On the algebraic side, this corresponds to studying $L$-graded rings $A=\oplus_{n \in L} A_{n}$.

\subsection{Coinvariants and the scheme of fixed points}

4.1.1 Coinvariants. Given an $L$-graded ring $A$, consider the ideal $I$ generated by all modules $A_{n}$ with $n \neq 0$. It is a graded ideal, and one has $I_{n}=A_{n}$ for $n \neq 0$ and $I_{0}=\sum_{n \neq 0} A_{n} A_{-n}$. Note that $A / I=A_{0} / I_{0}$ is the maximal graded quotient of $A$ with trivial $L$-grading. We call it the ring of coinvariants of $A$ and denote it $A_{G}$ or $A_{L}$.

4.1.2 The scheme of fixed points. If $X=\operatorname{Spec}(A)$, then $X^{G}:=\operatorname{Spec}\left(A_{G}\right)$ is the maximal closed subscheme of $X$ on which the action is trivial. Obviously, $X \mapsto X^{G}$ is a functor on the category of affine $G$ schemes. We call it the fixed-points functor; see also Section 5.1.13 below.

\subsection{Invariants and the quotient}

4.2.1 The definition. If $A$ is an $L$-graded ring, then $G=\mathbf{D}_{L}$ acts on $A$, and $A_{d}$ is the set of all elements on which $G$ acts through $\chi_{d}$. In particular, the ring of invariants $A^{G}$ coincides with $A_{0}$. The quotient of $X=\operatorname{Spec}(A)$ by the action is the scheme $X / / G:=\operatorname{Spec}\left(A_{0}\right)$. Obviously, $X \mapsto X / / G$ is a functor on the category of affine $G$ schemes. We call it the quotient functor.

4.2.2 Some properties preserved by the quotient functor. It is classical that the properties of being reduced, integral, and normal are preserved by quotients.

Lemma 4.2.3. Assume that an $L$-graded ring $A=\oplus_{n \in L} A_{n}$ satisfies one of the following properties: $A$ is a reduced ring, an integral domain, or a normal domain. Then $A_{0}$ satisfies the same property.

Proof. Only the last case needs justification. Assume that $A$ is a normal domain and so $A_{0}$ is a domain. If $a, b \in A_{0}$ are such that $b \neq 0$ and $c=a / b$ is integral over $A_{0}$, then $c \in A$. Since $b c=a$ in the domain $A$ and $a, b \in A_{0}$, we obtain that $c$ is also homogeneous of degree zero.

Another property preserved by quotients is being of finite type.

Lemma 4.2.4. Assume that an L-graded ring $A=\oplus_{n \in L} A_{n}$ is finitely generated over a subring $C \subseteq A_{0}$. Then $A_{0}$ is finitely generated over $C$.

Proof. We can choose homogeneous $C$-generators of $A$, and this gives a presentation of $A$ as a quotient of an $L$-graded polynomial algebra $B=C\left[t_{1}, \ldots, t_{l}\right]$ by a homogeneous ideal $I$. Then $A_{n}=B_{n} / I_{n}$ for any $n \in L$, and so $A_{0}$ is a quotient of $B_{0}$. Note that $B_{0}=C[M]$ for the monoid $M=\phi^{-1}(0)$ with $\phi: \mathbb{N}^{l} \rightarrow L$ given by $\phi\left(a_{1}, \ldots, a_{l}\right)=\sum_{i=1}^{l} a_{i} \operatorname{deg}\left(t_{i}\right)$. Since the monoid $M$ is finitely generated, $B_{0}$ is finitely generated over $C$, and the lemma follows.

4.2.5 The universality of quotients and fixed-point schemes. As opposed to the case of general reductive groups, the quotient is universal in the sense of [MFK94, Chapter 0, $\S 1$ ], regardless of the characteristic.

Lemma 4.2.6. Assume that $G=\mathbf{D}_{L}$ acts on $X=\operatorname{Spec}(A)$, write $Y=X / / G$, let $Y^{\prime} \rightarrow Y$ be an affine morphism, and let $X^{\prime}=Y^{\prime} \times_{Y} X$. Then $X^{\prime} / / G=Y^{\prime}$ and $\left(X^{\prime}\right)^{G}=X^{G} \times_{X} X^{\prime}$. 


\section{Abramovich and M. Temkin}

Proof. Let $Y^{\prime}=\operatorname{Spec}\left(B_{0}\right)$. Since $Y=\operatorname{Spec}\left(A_{0}\right)$, we obtain $X^{\prime}=\operatorname{Spec}(B)$, where $B=B_{0} \otimes_{A_{0}} A$ with the grading $B=\oplus_{n \in L} B_{0} \otimes_{A_{0}} A_{n}$. So, $B_{0}$ is the degree zero component of $B$, and hence $X^{\prime} / / G=Y^{\prime}$. In addition, $A_{n} B=B_{n} B$ for any $n \in L$, hence $B_{G}=B \otimes_{A} A_{G}$, giving

$$
\left(X^{\prime}\right)^{G}=\operatorname{Spec} B_{G}=\operatorname{Spec} B \otimes_{A} A_{G}=X^{G} \times_{X} X^{\prime} .
$$

Remark 4.2.7. A much more general result is proved by Alper in [Alp08, Proposition 4.7(i)] in the context of good moduli spaces.

4.2.8 Fibers. The quotient morphism $X \rightarrow Y=X / / G$ is $G$-equivariant with respect to the trivial action on $Y$; hence it contracts the orbits of the action on $X$. It is well known that two orbits of closed points are mapped to the same point if and only if their closures intersect, so on the set-theoretical level, one can view $Y$ as the "separated" quotient of $X$. For example, if $X$ is defined over a field, then the same fact is proved for any reductive group action in [MFK94, Corollary 1.2]. In the case of a diagonalizable group, one can obtain a more precise description as follows.

Lemma 4.2.9. Assume that $G=\mathbf{D}_{L}$ acts on $X=\operatorname{Spec}(A)$, and let $y$ be a point of $Y=X / / G$. Then the fiber $X_{y}$ contains a single orbit $O$ which is closed in $X_{y}$, and this orbit belongs to the closure of any other orbit contained in $X_{y}$.

Proof. By Lemma 4.2.6, we can replace $Y$ with $y=\operatorname{Spec}(k(y))$ and $X$ with $X_{y}=X \times_{Y} y$. So, we can assume that $Y=\operatorname{Spec}(k)$ for a field $k$ and $X=\operatorname{Spec}(A)$ for an $L$-graded $k$-algebra $A=\oplus_{n \in L} A_{n}$ with $A_{0}=k$. The set $L^{\prime}$ of elements $n \in L$ such that $A_{n}$ contains a unit of $A$ is a subgroup of $L$. Furthermore, if $n \in L^{\prime}$, then any non-zero element of $A_{n}$ is a unit because $A_{0}$ is a field. So, $I=\oplus_{n \in L \backslash L^{\prime}} A_{n}$ is the maximal homogeneous ideal of $A$. The closure of any orbit is given by a homogeneous ideal, hence contains $O=\operatorname{Spec}(A / I)$. It remains to observe that $O \stackrel{\sim}{\rightarrow} \operatorname{Spec}\left(k\left[L^{\prime}\right]\right)$ is a single orbit with stabilizer $\mathbf{D}_{L / L^{\prime}}$.

EXAmple 4.2.10. If $G=\mathbb{G}_{m}$, then there are two types of fibers: either $X_{y}$ contains a single $G$-invariant closed point, or $X_{y}$ is a single orbit with stabilizer $\mu_{n}$.

Recall that a morphism $X \rightarrow Y$ is submersive if $U \subset Y$ is open if and only if its preimage is open [SP17, tag/0406]. Lemmas 4.2.6 and 4.2.9 and [MFK94, $\S 0.2$, Remarks 5 and 6] imply the following result.

Corollary 4.2.11. If $G=\mathbf{D}_{L}$ acts on $X=\operatorname{Spec}(A)$, then $X / / G$ is a universal categorical quotient and the quotient morphism $X \rightarrow X / / G$ is submersive.

EXAMPLE 4.2.12. For completeness, we note that the above theory completely breaks down for non-reductive groups. The classical example is obtained when $X=\operatorname{Spec}(A)$ is $\mathrm{GL}_{2}(k)$ and $G$ is a Borel subgroup acting on $X$ on the left. Then $A^{G}=k$, but the categorical quotient is $\mathbb{P}_{k}^{1}$. In particular, $\operatorname{Spec}\left(A^{G}\right)$ is just the affine hull of the categorical quotient.

\subsection{Noetherian $L$-graded rings}

A theorem of Goto and Yamagishi states that any noetherian $L$-graded ring is finitely generated over the subring of invariants; see [GY83]. In the case of a finite group action (not necessarily commutative), an analogous claim was recently proved by Gabber; see [ILO14, Proposition IV.2.2.3]. It seems that the work [GY83] is not widely known in algebraic geometry; at least, we had reproved the theorem (in a more complicated way!) before finding the reference. For the sake of completeness, we outline the proof of [GY83, Theorem 1.1] below. 


\section{LUNA'S FUNDAMENTAL LEMMA FOR DIAGONALIZABLE GROUPS}

Proposition 4.3.1 (Goto-Yamagishi). Assume that $A=\oplus_{n \in L} A_{n}$ is a noetherian L-graded ring. Then $A_{0}$ is noetherian, each $A_{n}$ is a finitely generated $A_{0}$-module, and $A$ is a finitely generated $A_{0}$-algebra.

Proof. If $n \in L$, then for any $A_{0}$-submodule $M \subseteq A_{n}$, the ideal $M A$ of $A$ satisfies $M A \cap A_{n}=M$. It follows that each $A_{n}$ is a noetherian $A_{0}$-module, so $A_{0}$ is a noetherian ring and each $A_{0^{-}}$ module $A_{n}$ is finitely generated. It remains to prove that $A$ is finitely generated over $A_{0}$, and the case of a finite $L$ is, now, obvious.

In general, $L$ is a direct sum of cyclic groups, and using that $A^{L^{\prime} \oplus L^{\prime \prime}}=\left(A^{L^{\prime}}\right)^{L^{\prime \prime}}$, we reduce the claim to the case when $L$ is cyclic. Thus, we can assume $L=\mathbb{Z}$. It suffices to prove that $A_{\geqslant 0}=\oplus_{n \geqslant 0} A_{n}$ is finitely generated over $A_{0}$. Indeed, the same is then true for $A_{\leqslant 0}=\oplus_{n \leqslant 0} A_{n}$ by symmetry, and we win.

Set $A_{+}=\oplus_{n>0} A_{n}$. The homogeneous ideal $A_{+} A$ is finitely generated, so we can choose its homogeneous generators $f_{1}, \ldots, f_{l} \in A_{+}$. Note that $n_{i}=\operatorname{deg}\left(f_{i}\right)>0$. Set $n=\max _{i} n_{i}$, and let $C \subseteq A_{\geqslant 0}$ be the $A_{0}$-subalgebra generated by $A_{0}, \ldots, A_{n}$. We claim that $C=A_{\geqslant 0}$, so the latter is finitely generated over $A_{0}$. Indeed, by induction on $m>n$, we can assume that $A_{0}, \ldots, A_{m-1}$ lie in $C$. Any $g \in A_{m}$ can be represented as $\sum g_{i} f_{i}$, and the equality is preserved when we remove from each $g_{i}$ the components of degree different from $m-n_{i}$. But then $g_{i} \in A_{m-n_{i}} \subset C$ by the induction assumption, and so $g \in C$.

\section{4 $L$-local rings}

In this section, we study $L$-local rings that play the role of local rings among $L$-graded rings.

Remark 4.4.1. In fact, one can develop an $L$-graded analogue of commutative algebra (and algebraic geometry) which goes rather far. See [Tem04, §1], where graded versions of fields, local rings, fields of fractions, prime ideals, spectra, valuation rings, etc., are introduced. Many formulations and arguments are extended to the graded case just by replacing "elements" (of a ring or a module) with "homogeneous elements."

4.4.2 Maximal homogeneous ideals. By a maximal homogeneous ideal of an $L$-graded ring $A$, we mean any homogeneous ideal $m \subsetneq A$ such that no homogeneous ideal $n$ satisfies $m \subsetneq n \subsetneq A$. Note that $m$ does not have to be a maximal ideal of $A$.

4.4.3 Graded fields. An $L$-graded ring $k$ is called an $L$-graded field if 0 is the only proper homogeneous ideal of $A$. Equivalently, any non-zero homogeneous element of $A$ is invertible. Note that $m \subseteq A$ is a maximal homogeneous ideal if and only if $A / m$ is a graded field. Graded fields are analogues of fields in the category of graded rings. In particular, it is easy to see that any graded module over $k$ is a free $k$-module; see [Tem04, Lemma 1.2].

4.4.4 L-local rings. An $L$-graded ring $A$ that possesses a single maximal homogeneous ideal $m$ will be called $L$-local, and we will often use the notation $(A, m)$. A homogeneous homomorphism $\phi: A \rightarrow B$ of $L$-local rings is called $L$-local if it takes the maximal homogeneous ideal of $A$ to that of $B$. Here are a few other ways to characterize $L$-local rings.

Lemma 4.4.5. For an $L$-graded ring $A$, the following conditions are equivalent:

(i) The ring $A$ is L-local.

(ii) The ring $A_{0}$ is local. 


\section{Abramovich and M. Temkin}

(iii) The action of $\mathbf{D}_{L}$ on $\operatorname{Spec}(A)$ possesses a single closed orbit.

Proof. Homogeneous ideals of $A$ correspond to closed $\mathbf{D}_{L}$-equivariant subsets of $\operatorname{Spec}(A)$; hence statement (i) is equivalent to statement (iii). On the other hand, each fiber of the quotient map $\operatorname{Spec}(A) \rightarrow \operatorname{Spec}\left(A_{0}\right)$ contains a single closed orbit; hence statement (ii) is equivalent to statement (iii).

EXAmPle 4.4.6. A homogeneous ideal $p \subsetneq A$ is called $L$-prime in [Tem04, $\S 1$ ] if for any two homogeneous elements $x, y$ with $x y \in p$, at least one of them lies in $p$. Inverting all homogeneous elements in $A \backslash p$, one obtains an $L$-local ring $A_{p, L}$ that we call the homogeneous localization of $A$ at $p$. Even if $p$ is prime in the usual sense, $A_{p, L}$ is usually smaller than the usual localization $A_{p}$.

4.4.7 Homogeneous nilpotent radical. By the homogeneous nilpotent radical, we mean the ideal generated by all homogeneous nilpotent elements. The following result is proved precisely as its classical ungraded analogue.

Lemma 4.4.8. Let $A$ be an $L$-graded ring. Then the homogeneous nilpotent radical of $A$ coincides with the intersection of all $L$-prime ideals of $A$.

\subsubsection{Graded Nakayama's lemma.}

Proposition 4.4.10. Let $(A, m)$ be an $L$-local ring, and let $M$ be a finitely generated $L$-graded $A$-module. Then $m M=M$ if and only if $M=0$.

Proof. Note that $M=0$ if and only if its support is empty. By Nakayama's lemma, the latter consists of all points $x \in \operatorname{Spec}(A)$ such that $M(x)=M \otimes_{A} k(x) \neq 0$. Since the support is $\mathbf{D}_{L^{-}}$ equivariant, it is given by a homogeneous ideal, and hence it either is empty or contains $V(m)$. It follows that $M=0$ if and only if $M(x)=0$ for any $x \in V(m)$. The latter is equivalent to the vanishing of $M / m M$.

As in the usual situation, Nakayama's lemma has the following immediate corollary.

Corollary 4.4.11. Let $(A, m)$ be an $L$-local ring with residue graded field $k=A / m$, and let $M$ be a finitely generated $L$-graded $A$-module. Then:

(i) A homogeneous homomorphism of $L$-graded $A$-modules $\phi: N \rightarrow M$ is surjective if and only if $\phi \otimes_{A} k$ is surjective.

(ii) Homogeneous elements $m_{1}, \ldots, m_{l}$ generate $M$ if and only if their images generate $M / m M$.

(iii) The minimal cardinality of a set of homogeneous generators of $M$ equals the rank of the free $k$-module $M / m M$.

4.4.12 Equivariant Cartier divisors. As a corollary of the graded Nakayama's lemma, we can give the following characterization of equivariant divisors that will be used in [AT17] when toroidal actions are studied. (The finite presentation assumption is only essential in the nonnoetherian case.)

Proposition 4.4.13. Assume that $(A, m)$ is an L-local integral domain and $D \subset \operatorname{Spec}(A)$ is an equivariant, finitely presented closed subscheme. Let $x$ be an arbitrary point of $V(m)$, and let $X_{x}=\operatorname{Spec}\left(\mathcal{O}_{X, x}\right)$ be the localization at $x$. Then the following conditions are equivalent:

(i) We have $D=V(f)$ for a homogeneous element $f \in A$, 


\section{LUNA'S FUNDAMENTAL LEMMA FOR DIAGONALIZABLE GROUPS}

(ii) The scheme $D$ is a Cartier divisor in $X$,

(iii) The localization $D_{x}=D \times_{X} X_{x}$ is a Cartier divisor in $X_{x}$.

Proof. The only implication that requires a proof is (iii) $\Longrightarrow(\mathrm{i})$. By our assumptions, $D=V(I)$ for a finitely generated homogeneous ideal $I$, so $I / m I$ is a free $A / m$-module of a finite rank $d$. Since $D_{x}$ is Cartier, the $k(x)$-vector space $I \otimes_{A} k(x)=(I / m I) \otimes_{A} k(x)$ is one-dimensional, and we obtain $d=1$. Then $I$ is generated by a single homogeneous element $f$ by Corollary 4.4.11.

4.4.14 Regular parameters. Let $(A, m)$ be an $L$-local ring. Then $O=\operatorname{Spec}(A / m)$ is the closed orbit of $\operatorname{Spec}(A)$, and hence $A / m=k\left[L^{\prime}\right]$, where $k=A_{0} / m_{0}$ is the residue field of $A_{0}$ and $L^{\prime} \subseteq L$ is a subgroup.

LEMma 4.4.15. Keep the above notation and assume that $A$ is a regular ring and the torsion degree of $L^{\prime}$ is invertible in $k$. Let $n$ be the codimension of $O$ in $\operatorname{Spec}(A)$; then there exist homogeneous elements $t_{1}, \ldots, t_{n} \in A$ that generate $m$.

Proof. By Nakayama's lemma (Lemma 4.4.11(iii)), we should only check that the rank of the free $A / m$-module $m / m^{2}$ is $n$. Note that $m / m^{2}$ defines the conormal sheaf to $O$. But $O$ is regular by our assumption on the torsion of $L^{\prime}$, hence the rank of the conormal sheaf is $n$.

\subsection{Strictly $L$-local rings}

4.5.1 The definition. An $L$-local ring $(A, m)$ is called strictly $L$-local if $m$ contains any $A_{n}$ with $n \neq 0$. Here are a few natural ways to reformulate this.

Lemma 4.5.2. Let $(A, m)$ be an $L$-local ring, and let $m_{0}=m \cap A_{0}$. Then the following conditions are equivalent:

(i) The ring $A$ is strictly L-local.

(ii) The closed orbit of $\mathbf{D}_{L}$ on $\operatorname{Spec}(A)$ is a point.

(iii) $A / m=A_{0} / m_{0}$.

(iv) $m=m_{0} \oplus\left(\oplus_{0 \neq n \in L} A_{n}\right)$.

\subsubsection{Regularity and coinvariants.}

Lemma 4.5.4 ([Fog73, Corollary of Theorem 5.4]). If a strictly L-local ring $(A, m)$ is regular, then the ring of coinvariants $A_{L}$ is regular.

Proof. Choose $t_{1}, \ldots, t_{l}$ as in Lemma 4.4.15. Since $A / m$ is a field, they form a regular family of homogeneous parameters. First, consider the case when all $t_{i}$ are of degree zero. We claim that $A=A_{0}$, and so $A_{L}=A$ is regular. Indeed, we have $m=m_{0} A$, where $m_{0}=m \cap A_{0}$. Choose $0 \neq n \in L$. Since $A_{n} \subset m$, we obtain $m_{0} A_{n}=A_{n}$. But $A_{n}$ is a finitely generated $A_{0}$-module by Proposition 4.3.1, and so $A_{n}=0$ by Nakayama's lemma.

Now, assume that the degrees are arbitrary, and reorder the $t_{i}$ so that $t_{1}, \ldots, t_{q}$ are the only elements of degree zero. Then $A^{\prime}=A /\left(t_{q+1}, \ldots, t_{l}\right)$ is regular and we have $A_{L}^{\prime}=A_{L}$. It remains to observe that the images of $t_{1}, \ldots, t_{q}$ form a regular family of parameters of $A^{\prime}$, and so $A_{L}^{\prime}=A^{\prime}$ by the above case.

4.5.5 Completion. By an L-complete local ring, we mean a complete local ring $(A, m)$ provided with a formal L-grading $A=\prod_{n \in L} A_{n}$ such that $A_{n} \subset m$ for each $n \neq 0$. In particular, 


\section{Abramovich and M. Temkin}

$A$ is strictly $L$-local in the formal sense and its residue field is trivially graded. Homogeneous homomorphisms of $L$-complete local rings are defined in the obvious way. The main motivation for considering this class of rings is the following result.

Proposition 4.5.6. Assume that $L$ is a finitely generated abelian group and $(A, m)$ is a noetherian strictly $L$-local ring. Set $m_{0}=m \cap A_{0}$, and for each $n \in L$, let $\widehat{A}_{n}$ denote the $m_{0}$-adic completion of the $A_{0}$-module $A_{n}$. Then, the $m$-adic completion of $A$ is isomorphic to $\prod_{n \in L} \widehat{A}_{n}$; in particular, it is an $L$-complete local ring.

Proof. Write $m_{0}^{A}=m_{0} A$. We need to relate the $m$-adic and $m_{0}^{A}$-adic topologies on $A$. We have the following.

Lemma 4.5.7. (i) There exist a constant $b>0$ and, for every $n \in L$, a constant $r(n)>0$, such that the following holds. Let $a \in A_{n} \cap m^{N}$. If $N \geqslant b q+r(n)$, then $a \in\left(m_{0}^{A}\right)^{q}$.

(ii) For each $N$, the set $\left\{n \in L \mid A_{n} \not \subset m^{N}\right\}$ is finite.

Proof. (i) By Proposition 4.3.1, the ring $A$ is finitely generated over $A_{0}$; hence we can choose homogeneous $A_{0}$-generators $f_{1}, \ldots, f_{k} \in A \backslash A_{0}$. Denote their degrees by $n_{1}, \ldots, n_{k} \in L \backslash\{0\}$, and consider the monoid homomorphism $\phi: \mathbb{N}^{k} \rightarrow L$ sending the $i$ th generator to $n_{i}$. An element of $A_{n}$ is a polynomial in the $f_{i}$ whose monomials have exponents in $\phi^{-1}(n)$. Any monomial with exponent in $\phi^{-1}(0)$ lies in $m_{0}$, and the claim is proven if we find $b>0$ and $r(n)>0$ and show that each monomial appearing in $a \in A_{n} \cap m^{N}$ with $N \geqslant b q+r(n)$ is divisible by a product of $q$ monomials in $m_{0}$. Writing $\left|\left(l_{1}, \ldots, l_{k}\right)\right|=\sum l_{i}$, we need to find $b>0$ and $r(n)>0$ and show that each monomial $f_{1}^{l_{1}} \cdots f_{k}^{l_{k}}$ of degree $\left|\left(l_{1}, \ldots, l_{k}\right)\right|=N \geqslant b q+r(n)$ in $f_{i}$ such that $\phi\left(l_{1}, \ldots, l_{k}\right)=n$ is divisible by a product of $q$ monomials lying in $m_{0}$. This is a combinatorial question on lattices.

Consider first the case $n=0$. The monoid $\phi^{-1}(0)$ is finitely generated, $\phi^{-1}(0)=\left\langle g_{1}, \ldots, g_{s}\right\rangle$. Write $b=\max \left(\left|g_{i}\right|\right)$. If $\left(l_{1}, \ldots, l_{k}\right) \in \phi^{-1}(0)$, then it has an integer expression $\left(l_{1}, \ldots, l_{k}\right)=\sum q_{i} g_{i}$, and $q=\sum q_{i} \geqslant N / \max \left(\left|g_{i}\right|\right)$. So, $f_{1}^{l_{1}} \cdots f_{k}^{l_{k}}$ is the product of at least $q$ elements of the form $f^{g}$ in $m_{0}$. This gives the result in this case.

Consider the general case. Since the monoid $\mathbb{N}^{k}$ is noetherian, the ideal generated by $\phi^{-1}(n)$ has finitely many generators $h_{1}, \ldots, h_{t} \in \phi^{-1}(n)$. It follows that $\phi^{-1}(n)=\cup_{i}\left(h_{i}+\phi^{-1}(0)\right)$. Write $r=\max \left(\left|h_{i}\right|\right)$. Then any $\left(l_{1}, \ldots, l_{k}\right) \in \phi^{-1}(n)$ of degree $N \geqslant b q+r$ can be written as $h_{i}+t$, with $t \in \phi^{-1}(0)$ and $\operatorname{deg}(t) \geqslant b q$; hence $t$ is the sum of at least $q$ elements of $\phi^{-1}(0)$, which gives the general case.

(ii) Let $a \in A_{n} \subset A$ with $a \notin m^{N}$. Then any expression $a=\sum c_{j} f_{1}^{l_{j 1}} \cdots f_{k}^{l_{j k}}$ has at least one monomial of degree $\left|\left(l_{j 1}, \ldots, l_{j k}\right)\right|<N$. But the set of $\left(l_{1}, \ldots, l_{k}\right)$ of degree $<N$ is finite; therefore the set of $n=\phi\left(l_{1}, \ldots, l_{k}\right)$ with $\left|\left(l_{1}, \ldots, l_{k}\right)\right|<N$ is finite, as required.

Proof of Proposition 4.5.6, continued. Note that $\widehat{A}_{n}=\lim _{N} A_{n} /\left(m^{N} \cap A_{n}\right)$ since $m_{0}^{N} A_{n} \subset\left(m^{N} \cap\right.$ $\left.A_{n}\right) \subset m_{0}^{q(N)} A_{n}$ by Lemma 4.5.7(i). Also, claim (ii) of the same lemma implies that $\oplus_{n} A_{n} /\left(m^{N} \cap\right.$ $\left.A_{n}\right)=\prod_{n} A_{n} /\left(m^{N} \cap A_{n}\right)$. Using that products are compatible with limits, we obtain

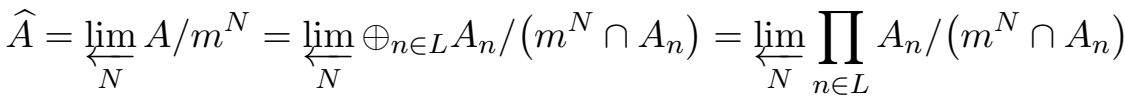

$$
\begin{aligned}
& =\prod_{n \in L} \lim _{\stackrel{N}{N}} A_{n} /\left(m^{N} \cap A_{n}\right)=\prod_{n \in L} \widehat{A}_{n}
\end{aligned}
$$

as required. 


\section{LUNA'S FUNDAMENTAL LEMMA FOR DIAGONALIZABLE GROUPS}

\subsection{Strongly homogeneous homomorphisms}

We say that a homogeneous homomorphism $\phi: A \rightarrow B$ of $L$-graded rings (respectively, $L$ complete local rings) is strongly homogeneous if $A \otimes_{A_{0}} B_{0}=B$ (respectively, $A \widehat{\otimes}_{A_{0}} B_{0}=B$ ) as $L$-graded rings (respectively, $L$-complete local rings). Similarly, if $P$ is a property of homomorphisms stable under base changes, for example smoothness, then we say that $\phi$ is strongly $P$ if it is strongly homogeneous and $\phi_{0}: A_{0} \rightarrow B_{0}$ satisfies $P$. In particular, $\phi$ satisfies $P$.

In addition, we will use the notion of strong formal smoothness, but only for local homomorphisms of strictly $L$-local rings. This makes sense because formal smoothness of local homomorphisms is preserved by base changes along local homomorphisms, and if $A$ is strictly $L$-local, then $A_{0} \rightarrow A$ is a local homomorphism.

Our next aim is to establish a criterion of strong homogeneity in one of the following two cases:

(1) the local case: $\phi$ is a local homomorphism of noetherian strictly $L$-local rings

(2) the formal case: $\phi$ is a local homomorphism of noetherian $L$-complete local rings

\subsubsection{Reduction to the formal case. First, let us reduce the problem to the formal case.}

Lemma 4.6.2. Assume that $\phi: A \rightarrow B$ is a homogeneous local homomorphism of noetherian strictly L-local rings. Then $\phi$ is strongly homogeneous if and only if its completion $\widehat{\phi}: \widehat{A} \rightarrow \widehat{B}$ is strongly homogeneous.

Proof. Clearly, $\phi$ is strongly homogeneous if and only if $f_{n}: A_{n} \otimes_{A_{0}} B_{0} \rightarrow B_{n}$ is an isomorphism for any $n \in L$, and it follows from Proposition 4.5.6 that $\widehat{\phi}$ is strongly homogeneous if and only if $g_{n}: \widehat{A}_{n} \widehat{\otimes}_{\widehat{A}_{0}} \widehat{B}_{0} \rightarrow \widehat{B}_{n}$ is an isomorphism for any $n \in L$. Since $B_{n}$ is finitely generated over $B_{0}$, we have $\widehat{B}_{n}=B_{n} \otimes_{B_{0}} \widehat{B}_{0}$, and since $A_{n}$ is finitely generated over $A_{0}$ we have $\widehat{A}_{n} \widehat{\otimes}_{\widehat{A}_{0}} \widehat{B}_{0}=A_{n} \otimes_{A_{0}} \widehat{B}_{0}$. Thus, $g_{n}: A_{n} \otimes_{A_{0}} \widehat{B}_{0} \rightarrow B_{n} \otimes_{B_{0}} \widehat{B}_{0}$ is the base change of $f_{n}$ with respect to the faithfully flat homomorphism $B_{0} \rightarrow \widehat{B}_{0}$, and hence $f_{n}$ is an isomorphism if and only $g_{n}$ is an isomorphism. The lemma follows.

4.6.3 The fiber. Let $\phi$ be as in case (1) or (2). Set $m=m_{A}$ and $k=A / m$, and consider the fiber $\bar{\phi}: k \rightarrow \Lambda=B / m B$ of $\phi$. If $\phi$ is strongly homogeneous, then the fiber is strongly homogeneous too, and since $k$ is trivially graded, we obtain that $\Lambda$ is trivially graded too. The geometric meaning of the latter condition is that the action of $\mathbf{D}_{L}$ on the fiber $\operatorname{Spec}(\Lambda)$ is trivial.

LEMma 4.6.4. Let $k$ be a trivially graded field and $\Lambda$ a noetherian local $k$-algebra with residue field $l=\Lambda / m_{\Lambda}$ and an $L$-grading making the structure homomorphism $\psi: k \rightarrow \Lambda$ homogeneous. Provide the $l$-vector spaces $\Omega_{\Lambda / k} \otimes_{\Lambda} l$ and $m_{\Lambda} / m_{\Lambda}^{2}$ with the induced $L$-grading. Then the following conditions are equivalent:

(i) The $k$-algebra $\Lambda$ is trivially graded.

(ii) The field $l$ and the space $m_{\Lambda} / m_{\Lambda}^{2}$ are trivially graded.

(iii) The field $l$ and the space $\Omega_{\Lambda / k} \otimes_{\Lambda} l$ are trivially graded.

Proof. Clearly, condition (i) implies condition (ii). Conversely, if condition (ii) holds, then each vector space $m_{\Lambda}^{n} / m_{\Lambda}^{n+1}$ is trivially graded. Since $\cap_{n} m_{\Lambda}^{n}=0$ by the noetherian assumption, it follows that $\Lambda$ is trivially graded. 


\section{Abramovich and M. Temkin}

To prove the equivalence of conditions (ii) and (iii), we observe that the homomorphisms $k \rightarrow \Lambda \rightarrow l$ induce an exact triangle of cotangent complexes - the transitivity triangle [Ill71, II.2.1.2.1] — whose associated exact sequence of homologies contains

$$
\Upsilon_{l / k} \rightarrow m_{\Lambda} / m_{\Lambda}^{2} \rightarrow \Omega_{\Lambda / k} \otimes_{\Lambda} l \rightarrow \Omega_{l / k}
$$

Both in conditions (ii) and (iii), the field $l$ is trivially graded, and hence the end terms $\Upsilon_{l / k}$ and $\Omega_{l / k}$ are trivially graded. Thus, $m_{\Lambda} / m_{\Lambda}^{2}$ is trivially graded if and only if $\Omega_{\Lambda / k}$ is trivially graded, as needed.

Remark 4.6.5. The assumption on the grading of $l$ is automatic when $\Lambda$ is strictly $L$-local (as will be in all our applications), but it cannot be omitted in general. For example, consider the case when $k=\mathbb{R}$ and $\Lambda=l=\mathbb{C}=\mathbb{R} \oplus i \mathbb{R}$ as a $\mathbb{Z} / 2 \mathbb{Z}$-graded field.

4.6.6 Lifting a formal grading. The following lemma will be our main tool in studying homomorphisms with trivially graded fiber.

Lemma 4.6.7. Let $\phi: A \rightarrow B$ be a homogeneous local homomorphism of $L$-complete noetherian local rings with a trivially graded fiber $k \rightarrow \bar{B}=B / m_{A} B$. Assume that $g: A \rightarrow D$ and $h: D \rightarrow B$ form a formally smooth factorization of $\phi$ (Section 2.1.4). Then one can provide $D$ with a formal grading such that the following conditions hold:

(i) Both $g$ and $h$ are homogeneous homomorphisms.

(ii) The fiber $\bar{D}=D / m_{A} D$ is trivially graded.

(iii) The homomorphism $g$ is a graded base change of the trivially graded homomorphism $C(k) \rightarrow C(\bar{D})$ introduced in Section 2.2.10.

Proof. Since $\bar{B}$ is trivially graded, we have $\bar{B}=B_{0} / m_{0} B_{0}$, where $m_{0}$ is the trivially graded component of $m_{A}$. Fix a ring of coefficients $i: C(k) \rightarrow A_{0}$, so that all rings we consider become $C(k)$-algebras. In particular, we obtain homomorphisms $i_{B_{0}}: C(k) \rightarrow B_{0}$ and $i_{D}: C(K) \rightarrow D$ which we utilize below.

Recall that the homomorphism $C(\bar{g}): C(k) \rightarrow C(\bar{D})$ discussed in Section 2.2.10 is formally smooth; considering the composed homomorphism $C(\bar{D}) \rightarrow \bar{D} \rightarrow \bar{B}$ and the diagram

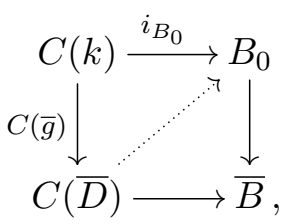

we can lift $C(\bar{D}) \rightarrow \bar{B}$ to a $C(k)$-homomorphism $C(\bar{D}) \rightarrow B_{0}$.

We claim that the composed homomorphism $\lambda: C(\bar{D}) \rightarrow B_{0} \rightarrow B$ lifts to a homomorphism $C(\bar{D}) \rightarrow D$ such that the composition $C(\bar{D}) \rightarrow D \rightarrow \bar{D}$ is the canonical projection $p: C(\bar{D}) \rightarrow \bar{D}$. Indeed, we have natural homomorphisms $(\lambda, p): C(\bar{D}) \rightarrow B \times{ }_{\bar{B}} \bar{D}$ and $D \rightarrow B \times \bar{B} \bar{D}$, giving a commutative diagram

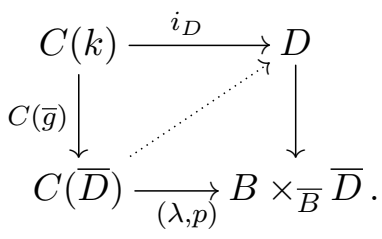




\section{LUNA'S FUNDAMENTAL LEMMA FOR DIAGONALIZABLE GROUPS}

By the Chinese remainder theorem, the homomorphism $D \rightarrow B \times{ }_{\bar{B}} \bar{D}$ is surjective, and using the formal smoothness of $C(\bar{g})$ again, we can lift $(\lambda, p)$ to a homomorphism $C(\bar{D}) \rightarrow D$.

At this stage, Theorem 2.2.11(ii), applied to the formally smooth homomorphism $g: A \rightarrow D$, asserts that $D=A \widehat{\otimes}_{C(k)} C(\bar{D})$, making $g$ the base change of $C(k) \rightarrow C(\bar{D})$. Provide $C(\bar{D})$ with the trivial grading. This induces the trivial grading on $\bar{D}$, giving property (ii). The formal grading $A=\prod_{n \in L} A_{n}$ provides a formal grading $D=\prod_{n \in L} A_{n} \widehat{\otimes}_{C(k)} C(\bar{D})$ making $g$ a graded base change, giving property (iii); in particular, $g$ is homogeneous. The homomorphism $C(\bar{D}) \rightarrow B$ is homogeneous since it factors through $B_{0}$. This implies that $A_{n} \widehat{\otimes}_{C(k)} C(\bar{D}) \rightarrow B$ factors through $A_{n} B_{0} \subset B_{n}$, so $h$ is homogeneous, showing property (i), as needed.

4.6.8 Strong formal smoothness. Already the case $D=B$ of the above lemma allows us to completely describe strongly formally smooth homomorphism (as defined in Section 4.6).

Corollary 4.6.9. Assume that $\phi: A \rightarrow B$ is a homogeneous local homomorphism of $L$-complete noetherian local rings. Then:

(i) The homomorphism $\phi$ is strongly formally smooth if and only if it is formally smooth and has a trivially graded fiber.

(ii) If $\phi$ is formally smooth, then the following conditions are equivalent: (a) $\phi$ has a trivially graded fiber, (b) $\phi$ is strongly homogeneous, (c) $\phi$ is strongly formally smooth.

Proof. The forward direction in statement (i) follows from the discussion in Section 4.6.3, so we assume that $\phi$ is formally smooth with trivially graded fiber and prove that $\phi$ is strongly homogeneous and $\phi_{0}$ is formally smooth. Applying Lemma 4.6.7 with $D=B$, we obtain $B=$ $A \widehat{\otimes}_{C(k)} C(\bar{B})$, where $C(k)$ and $C(\bar{B})$ are trivially graded. In particular, $B_{0}=A_{0} \widehat{\otimes}_{C(k)} C(\bar{B})$, and hence $\phi$ is strongly homogeneous. Moreover, $\phi_{0}$ is a base change of the formally smooth homomorphism $C(k) \rightarrow C(\bar{B})$; hence $\phi_{0}$ is formally smooth and we obtain statement (i).

To prove statement (ii), we note that the implications $(\mathrm{c}) \Longrightarrow(\mathrm{b}) \Longrightarrow(\mathrm{a})$ are obvious, and the implication $(\mathrm{a}) \Longrightarrow$ (c) follows from statement (i).

4.6.10 Strong homogeneity and the cotangent complex. Now we will study general factorizable homomorphisms; see Section 2.1.4. In this case, one should also control the grading of the kernel of a factorization $D \rightarrow B$. Naturally, this is related to the first homology of the cotangent complex $\mathbb{L}_{B / A}$.

Lemma 4.6.11. Assume that $g: A \rightarrow D$ is a formally smooth homogeneous local homomorphism of $L$-complete noetherian local rings with a trivially graded fiber $k \rightarrow \bar{D}$, let $I \subset D$ be a homogeneous ideal, $B=D / I$, and $l$ the residue field of $B$. Then the following conditions are equivalent:

(i) The composition $A \rightarrow B$ is strongly homogeneous.

(ii) The ideal $I$ is generated by elements of degree zero.

(iii) The module $\left(I / I^{2}\right) \otimes_{B} l$ is trivially graded.

(iv) The homology group $H_{1}\left(\mathbb{L}_{B / A} \otimes_{B}^{\mathrm{L}} l\right)$ is trivially graded.

Proof. (i) $\Longleftrightarrow$ (ii) The homomorphism $A \rightarrow D$ is strongly homogeneous by Corollary 4.6.9(i); hence $A \rightarrow B$ is strongly homogeneous if and only if $D \rightarrow B$ is strongly homogeneous. The latter happens if and only if $D / I=B=D \otimes_{D_{0}} B_{0}=D / I_{0} D$, where $I_{0}$ is the trivially graded part of $I$, that is, if and only if condition (ii) holds. 


\section{Abramovich and M. Temkin}

(ii) $\Longleftrightarrow$ (iii) The direct implication is clear. Conversely, assume that condition (iii) holds; then the $B$-module $I / I^{2}$ is generated by elements of degree zero by the graded Nakayama's lemma; see Corollary 4.4.11(ii). Since elements of $I$ generating $I / I^{2}$ generate $I$ by the usual Nakayama's lemma, we obtain condition (ii).

(iii) $\Longleftrightarrow$ (iv) Consider the exact triangle $\Delta$ obtained from the transitivity triangle

$$
\mathbb{L}_{D / A} \otimes_{D}^{\mathrm{L}} B \rightarrow \mathbb{L}_{B / A} \rightarrow \mathbb{L}_{B / D} \rightarrow \mathbb{L}_{D / A} \otimes_{D}^{\mathrm{L}} B[1]
$$

by applying $\cdot \otimes_{B}^{\mathrm{L}} l$. By the formal smoothness of $A \rightarrow D$, the complex $\mathbb{L}_{D / A} \otimes_{D}^{\mathrm{L}} l$ is quasiisomorphic to $\Omega_{D / A} \otimes_{D} l$. By [Ill71, III.1.2.8.1], we have $H_{0}\left(\mathbb{L}_{B / D}\right)=0$ and $H_{1}\left(\mathbb{L}_{B / D}\right)=I / I^{2}$; therefore $H_{1}\left(\mathbb{L}_{B / D} \otimes_{B}^{\mathrm{L}} l\right)=\left(I / I^{2}\right) \otimes_{B} l$. It follows that the exact sequence of homologies associated with $\Delta$ contains the sequence

$$
0 \rightarrow H_{1}\left(\mathbb{L}_{B / A} \otimes_{B}^{\mathrm{L}} l\right) \rightarrow\left(I / I^{2}\right) \otimes_{B} l \rightarrow \Omega_{D / A} \otimes_{D} l .
$$

By our assumption on $A \rightarrow D$, the term $\Omega_{D / A} \otimes_{D} l$ is trivially graded; hence $\left(I / I^{2}\right) \otimes_{B} l$ is trivially graded if and only if $H_{1}\left(\mathbb{L}_{B / A} \otimes_{B}^{\mathrm{L}} l\right)$ is trivially graded.

4.6.12 The main result: formal factorizable case. We summarize what we have done so far in the factorizable case. This will later be generalized to arbitrary homomorphisms, see Theorem 4.7.8, but our result here is slightly more precise since we use only formally smooth factorizations in part (ii).

Theorem 4.6.13. Assume that $\phi: A \rightarrow B$ is a formally factorizable homogeneous local homomorphism of $L$-complete noetherian local rings. Then the following conditions are equivalent:

(i) The homomorphism $\phi$ is strongly homogeneous.

(ii) The homomorphism $\phi$ factors into a composition of homogeneous homomorphisms $A \rightarrow$ $D \rightarrow D / I=B$, where $D$ is $L$-complete, $A \rightarrow D$ is formally smooth with a trivially graded fiber, and $I$ is generated by elements of degree zero.

(iii) The $l$-vector spaces $H_{0}\left(\mathbb{L}_{B / A} \otimes_{B}^{\mathrm{L}} l\right)=\Omega_{B / A} \otimes_{B} l$ and $H_{1}\left(\mathbb{L}_{B / A} \otimes_{B}^{\mathrm{L}} l\right)$ are trivially graded.

Proof. By Lemma 4.6.4, the homomorphism $\phi$ has a trivially graded fiber if and only if $\Omega_{B / A} \otimes_{B} l$ is trivially graded. This means that any of the conditions (i), (ii), and (iii) imply both that $\phi$ has a trivially graded fiber and that $\Omega_{B / A} \otimes_{B} l$ is trivially graded.

Fix a factorization $A \rightarrow D \rightarrow B$ with a formally smooth $A \rightarrow D$. Since $\phi$ has trivially graded fiber, we may apply Lemma 4.6.7: in case (i) or (iii), we may grade $D$ so that $A \rightarrow D$ and $D \rightarrow B$ become homogeneous and with a trivially graded fiber; this holds by assumption in case (ii).

It follows that under any of the assumptions (i), (ii), and (iii), the setup of Lemma 4.6.11 holds. Furthermore, assumption (i) is equivalent to Lemma 4.6.11(i), assumption (ii) is equivalent to Lemma 4.6.11(ii), and assumption (iii) is equivalent to Lemma 4.6.11(iv). The equivalence of assumptions (i), (ii), and (iii) now follows from the equivalence in Lemma 4.6.11.

Remark 4.6.14. Let $\phi: A \rightarrow B$ be a local homomorphism of $L$-complete noetherian local rings, and assume that the extension of the residue fields $l / k$ is separable (in particular, $\phi$ is factorizable). In this case, one can describe $\phi$ very explicitly.

Fix a ring of coefficients $C(k) \rightarrow A$. If $\phi$ is formally smooth, then by Remark 2.2.12, there exists an isomorphism of $A$-algebras $A \widehat{\otimes}_{C(k)} C(l) \llbracket t_{1}, \ldots, t_{n} \rrbracket \stackrel{\sim}{\rightarrow} B$. It follows that, in general, $\phi$ is strongly homogeneous if and only if there exists a homogeneous isomorphism of $A$-algebras

$$
\left(A \widehat{\otimes}_{C(k)} C(l) \llbracket t_{1}, \ldots, t_{n} \rrbracket\right) /\left(f_{1}, \ldots, f_{m}\right) \stackrel{\sim}{\rightarrow} B,
$$




\section{LUNA'S FUNDAMENTAL LEMMA FOR DIAGONALIZABLE GROUPS}

where $t_{i}$ and $f_{j}$ are of degree zero.

\subsection{Elimination of the formal factorization assumption}

The aim of this section is to extend the main results of Section 4.6 to non-factorizable homomorphisms. This only requires us to replace formally smooth factorizations by so-called Cohen factorizations. The arguments are very similar, so we will mainly indicate the modifications one has to make.

4.7.1 Cohen factorization. Let $\phi: A \rightarrow B$ be a homomorphism of noetherian complete local rings. A Cohen factorization of $\phi$ is a factorization of the form $A \stackrel{f}{\rightarrow} D \stackrel{g}{\rightarrow} B$, where $D$ is a noetherian complete local ring, $g$ is surjective, $f$ is flat, and the ring $D / m_{A} D$ is regular.

Remark 4.7.2. (i) Cohen factorizations were introduced by Avramov, Foxby, and Herzog in [AFH94] to study local homomorphisms between noetherian complete local rings. In particular, they showed that such a factorization always exists. Later, this notion was exploited by Avramov in [Avr99] to establish foundational properties of general lci morphisms.

(ii) For the sake of comparison with the formally smooth factorizations, recall that $f$ is formally smooth if it is flat and $D / m_{A} D$ is geometrically regular over $A / m_{A}$. Flat morphisms with regular fibers are sometimes called weakly regular, but they are not especially useful. So it may be surprising that Cohen factorizations do provide a useful tool.

4.7.3 Graded Cohen factorization. The following result will serve as a replacement of Lemma 4.6.7.

LEMma 4.7.4. Let $\phi: A \rightarrow B$ be a homogeneous local homomorphism of $L$-complete noetherian local rings with a trivially graded fiber $k \rightarrow \bar{B}=B / m_{A} B$. Then there exists a Cohen factorization $A \stackrel{g}{\rightarrow} D \stackrel{h}{\rightarrow} B$ such that the following conditions hold:

(i) Both $g$ and $h$ are homogeneous homomorphisms.

(ii) The homomorphism $g$ is a graded formal base change of a homomorphism $\psi: C(k) \rightarrow$ $C(l) \llbracket y_{1}, \ldots, y_{n} \rrbracket$, where $l=B / m_{B}$ and the gradings are trivial. In particular, the fiber $\bar{D}=$ $D / m_{A} D$ is trivially graded.

We note that, unlike the situation in Remark 4.6.14, the homomorphism $\psi$ does not take $C(k)$ to $C(l)$ in general.

Proof. The proof is a graded variation on the proof of [AFH94, Theorem 1.1], and it is also close to the proof of Lemma 4.6.7, so we only describe the construction. The rings $A$ and $B$ have the same residue fields as $A_{0}$ and $B_{0}$, respectively, so we can fix structure homomorphisms $C(k) \rightarrow A_{0}$ and $C(l) \rightarrow B_{0}$. Choose a surjective homomorphism $C(l) \llbracket y_{1}, \ldots, y_{n} \rrbracket \rightarrow B_{0}$ that takes $y_{i}$ to a family of generators of $m_{B_{0}}$; then the composed homomorphism $C(k) \rightarrow A_{0} \rightarrow B_{0}$ lifts to a homomorphism $\psi: C(k) \rightarrow C(l) \llbracket y_{1}, \ldots, y_{n} \rrbracket$. We provide $\psi$ with the trivial grading and define $g$ to be the graded formal base change $A \rightarrow D=A \widehat{\otimes}_{C(k)} C(l) \llbracket y_{1}, \ldots, y_{n} \rrbracket$. We claim that $g$ satisfies condition (ii). Indeed, $g$ is flat because $\psi$ and the completion homomorphism $A \otimes_{C(k)} C(l) \llbracket y_{1}, \ldots, y_{n} \rrbracket \rightarrow D$ are flat, and $D / m_{A} D=l \llbracket y_{1}, \ldots, y_{n} \rrbracket$ is regular.

It remains to construct $h$. The graded homomorphisms $C(l) \llbracket y_{1}, \ldots, y_{n} \rrbracket \rightarrow B_{0} \rightarrow B$ and $\phi$ induce a homomorphism $h: D \rightarrow B$, so we should only check that $h$ is onto. Since $D / m_{D}=l$, 


\section{Abramovich and M. Temkin}

we should only prove that $h(D)$ contains a set of generators of $m_{B}$. Clearly, $h(D)$ contains $m_{A} B$ and $m_{B_{0}}$, and it remains to note that since $B / m_{A} B$ is trivially graded, $m_{B}=m_{A} B+m_{B_{0}}$.

4.7.5 The cotangent complex. Next, we adjust Lemma 4.6.11 to Cohen factorizations.

Lemma 4.7.6. Assume that $g: A \rightarrow D$ is a graded formal base change of a trivially graded homomorphism $\psi: C(k) \rightarrow C(l) \llbracket y_{1}, \ldots, y_{n} \rrbracket$, where $k=A / m_{A}$ and $l=D / m_{D}$. If $I \subset D$ is a homogeneous ideal and $B=D / I$, then the following conditions are equivalent:

(i) The composition $A \rightarrow B$ is strongly homogeneous.

(ii) The ideal $I$ is generated by elements of degree zero.

(iii) The module $\left(I / I^{2}\right) \otimes_{B} l$ is trivially graded.

(iv) The homology group $H_{1}\left(\mathbb{L}_{B / A} \otimes_{B}^{\mathrm{L}} l\right)$ is trivially graded.

Proof. The proof is a copy of the proof of Lemma 4.6 .11 with the only difference that in the exact sequence

$$
H_{1}\left(\mathbb{L}_{D / A} \otimes_{D}^{\mathrm{L}} l\right) \rightarrow H_{1}\left(\mathbb{L}_{B / A} \otimes_{B}^{\mathrm{L}} l\right) \rightarrow\left(I / I^{2}\right) \otimes_{B} l \rightarrow \Omega_{D / A} \otimes_{D} l,
$$

the first term can be non-zero. However, we claim that it is trivially graded, and hence it is still true that $\left(I / I^{2}\right) \otimes_{B} l$ is trivially graded if and only if $H_{1}\left(\mathbb{L}_{B / A} \otimes_{B}^{\mathrm{L}} l\right)$ is trivially graded.

It remains to study $H_{1}\left(\mathbb{L}_{D / A} \otimes_{D}^{\mathrm{L}} l\right)$. Set $E=C(l) \llbracket y_{1}, \ldots, y_{n} \rrbracket$. Since $\psi$ is flat, the homomorphisms $\psi$ and $C(k) \rightarrow A$ are Tor-independent, and hence $\mathbb{L}_{D / A} \stackrel{\sim}{\rightarrow} \mathbb{L}_{E / C(k)} \otimes_{E}^{\mathrm{L}} D$ by [Ill71, Corollary III.2.2.3]. Thus, $\mathbb{L}_{D / A} \otimes_{D}^{\mathrm{L}} l=\mathbb{L}_{E / C(k)} \otimes_{E}^{\mathrm{L}} l$ is trivially graded since $\psi$ is trivially graded.

4.7.7 The main result: formal case. Now, we can eliminate the formal factorization assumption from our main formal result.

Theorem 4.7.8. Assume that $\phi: A \rightarrow B$ is a homogeneous local homomorphism of L-complete noetherian local rings. Then the following conditions are equivalent:

(i) The homomorphism $\phi$ is strongly homogeneous.

(ii) The homomorphism $\phi$ possesses a Cohen factorization $A \stackrel{g}{\rightarrow} D \rightarrow D / I=B$, where $g$ is a graded formal base change of a trivially graded homomorphism $\psi: C(k) \rightarrow C(l) \llbracket y_{1}, \ldots, y_{n} \rrbracket$ and $I$ is generated by elements of degree zero.

(iii) The l-vector spaces $H_{0}\left(\mathbb{L}_{B / A} \otimes_{B}^{\mathrm{L}} l\right)=\Omega_{B / A} \otimes_{B} l$ and $H_{1}\left(\mathbb{L}_{B / A} \otimes_{B}^{\mathrm{L}} l\right)$ are trivially graded.

Proof. The proof of Theorem 4.6.13 applies, with Lemmas 4.6.4 and 4.6.11 replaced by Lemmas 4.7.4 and 4.7.6.

4.7.9 Descent of lci. The notion of general lci morphisms between noetherian schemes was introduced by Avramov; see [Avr99, Section 1, Definition, pp. 458-459]. This is equivalent to the following: a local homomorphism of noetherian local rings $\phi: A \rightarrow B$ is called complete intersection if its completion possesses a Cohen factorization $\widehat{A} \rightarrow D \rightarrow \widehat{B}$ such that the kernel of $D \rightarrow \widehat{B}$ is generated by a regular sequence. This turns out to be independent of the factorization. A morphism of locally noetherian schemes is lci if all its local homomorphisms are complete intersections. In particular, $\phi$ is complete intersection if and only if it is lci, and we will use the notion lci from here on. 


\section{LUNA'S FUNDAMENTAL LEMMA FOR DIAGONALIZABLE GROUPS}

Recall that a flat lci morphism is called syntomic. Traditionally, one assumes a syntomic morphism to be locally of finite presentation, but with Avramov's general notion of lci morphisms, this assumption becomes redundant. Unlike general lci morphisms, syntomic morphisms are preserved by arbitrary base changes, so the notion of being strongly syntomic makes sense (as defined in Section 4.6).

Lemma 4.7.10. Let $\phi: A \rightarrow B$ be a homogeneous local homomorphism of $L$-complete noetherian local rings.

(i) Assume that $\phi$ is strongly homogeneous. If $\phi$ is lci, then $\phi_{0}: A_{0} \rightarrow B_{0}$ is lci. Conversely, if $\phi_{0}$ is lci and the homomorphisms $\phi_{0}$ and $A_{0} \hookrightarrow A$ are Tor-independent, then $\phi$ is lci.

(ii) The homomorphism $\phi$ is strongly syntomic if and only if it is syntomic and strongly homogeneous.

Proof. The implication (i) $\Longrightarrow$ (ii) is obvious, so let us prove part (i). The inverse implication is clear since lci morphisms are preserved by Tor-independent base changes and compositions, and the completion homomorphism $A \otimes_{A_{0}} B_{0} \rightarrow A \widehat{\otimes}_{A_{0}} B_{0}=B$ is lci.

Assume now that $\phi$ is lci. Consider a Cohen factorization $A \rightarrow D \rightarrow D / I=B$ as in Theorem 4.7.8. Then $I$ is generated by a regular sequence of length $n$, and hence any generating sequence of $I$ of size $n$ is a regular sequence. Note that $n$ is the dimension of $\left(I / I^{2}\right) \otimes_{D} l$, where $l=D / m_{D}$. By Lemma 4.7.6, the module $\left(I / I^{2}\right) \otimes_{D} l$ is trivially graded; hence we can find $x_{1}, \ldots, x_{n}$ of degree zero whose images generate $\left(I / I^{2}\right) \otimes_{D} l$. By Nakayama's lemma, the $x_{i}$ generate $I$. As we noted above, the $x_{i}$ form a regular sequence in $D$ and hence also in $D_{0}$. It remains to note that $A_{0} \rightarrow D_{0}$ is a formal base change of $C(k) \rightarrow C(l) \llbracket y_{1}, \ldots, y_{m} \rrbracket$, and hence $A_{0} \rightarrow D_{0} \rightarrow B_{0}$ is a Cohen factorization of $\phi_{0}$, and that the kernel of $D_{0} \rightarrow B_{0}$ coincides with $I_{0}$ and hence is generated by the regular sequence $x_{1}, \ldots, x_{n}$.

Remark 4.7.11. It may happen that $\phi$ is strongly homogeneous and $\phi_{0}$ is lci but $\phi$ is not lci. For example, take $A_{0}=k \llbracket x \rrbracket$ and $B_{0}=A_{0} /(x)=k$. Extend $A_{0}$ to a graded algebra $A=$ $A_{0}[\varepsilon] /\left(\varepsilon^{2}, x \varepsilon\right)$, where $\varepsilon$ is homogeneous of a non-zero degree. Finally, set $B=A \widehat{\otimes}_{A_{0}} B_{0}=A / x A=$ $k[\varepsilon] /\left(\varepsilon^{2}\right)$. Then $\phi: A \rightarrow B$ is strongly homogeneous but not lci since $x \varepsilon=0$ and hence $\{x\}$ is not a regular sequence.

\subsection{The strictly local Luna's fundamental lemma}

We complete our study of strong homogeneity with a summary of the strictly local case. Section 5 is devoted to the global relatively affine case.

Theorem 4.8.1. Assume that $\phi: A \rightarrow B$ is a homogeneous local homomorphism of noetherian strictly L-local rings. Then:

(i) The homomorphism $\phi$ is strongly homogeneous if and only if the l-vector spaces $H_{0}\left(\mathbb{L}_{B / A} \otimes \mathrm{L}_{B}\right.$ $l)=\Omega_{B / A} \otimes_{B} l$ and $H_{1}\left(\mathbb{L}_{B / A} \otimes_{B}^{\mathrm{L}} l\right)$ are trivially graded.

(ii) Assume that $\phi$ is formally smooth; then the following conditions are equivalent: (a) the $l$-vector space $\Omega_{B / A} \otimes_{B} l$ is trivially graded, (b) $\phi$ has a trivially graded fiber, (c) $\phi$ is strongly homogeneous, (d) $\phi$ is strongly formally smooth.

(iii) The homomorphism $\phi$ is strongly syntomic if and only if it is syntomic and strongly homogeneous. Moreover, if $\phi$ is strongly homogeneous, then the following claims hold: (a) if $\phi$ is lci, then $\phi_{0}$ is lci, (b) if $\phi_{0}$ is lci and Tor-independent with the homomorphism $A_{0} \rightarrow A$, then $\phi$ is lci. 


\section{Abramovich and M. TEmkin}

Proof. Recall that by Lemma 4.6.2, the homomorphism $\phi$ is strongly homogeneous if and only if its completion $\widehat{\phi}: \widehat{A} \rightarrow \widehat{B}$ is so. Since $H_{i}\left(\mathbb{L}_{B / A} \otimes_{B}^{\mathrm{L}} l\right)=H_{i}\left(\mathbb{L}_{\widehat{B} / \widehat{A}} \otimes_{\widehat{B}}^{\mathrm{L}} l\right)$ by [FR93, Lemma 1], claim (i) follows from Theorem 4.6.13.

Parts (a) and (b) of claim (ii) are equivalent by Lemma 4.6.4. The equivalence of parts (b), (c), and (d) reduces to Corollary 4.6.9(ii) because $\phi_{0}: A_{0} \rightarrow B_{0}$ is formally smooth if and only if its completion is formally smooth, and the latter coincides with the degree zero part of $\widehat{\phi}$ by Proposition 4.5.6.

Note that $\phi$ is lci or syntomic if and only if $\widehat{\phi}$ is so. Since $(\widehat{\phi})_{0}$ is the completion of $\phi_{0}$ by Proposition 4.5.6, it suffices to prove claim (iii) for $\widehat{\phi}$, and this has already been done in Lemma 4.7.10.

\section{Luna's fundamental lemma}

In Section 5, we study relatively affine actions of diagonalizable groups on general noetherian schemes and extend the classical Luna's fundamental lemma to this case. To simplify the exposition, we work with split groups and indicate in Section 5.7 how the non-split case can be deduced.

\subsection{Relatively affine actions}

5.1.1 The definition. An action of $G=\mathbf{D}_{L}$ on a scheme $X$ is called relatively affine if there exists a scheme $Z$ provided with the trivial $G$-action and an affine $G$-equivariant morphism $f: X \rightarrow Z$. In this case, we define the quotient $X / / G=\operatorname{Spec}_{Z}\left(f_{*}\left(\mathcal{O}_{X}\right)^{G}\right)$. We omit $Z$ in the notation because the quotient is categorical by the following theorem, and hence it is independent of the scheme $Z$. By definition, if $Y=X / / G$ is covered by affine open subschemes $Y_{i}$, then the $X_{i}=Y_{i} \times_{Y} X$ form an open affine equivariant covering of $X$ and $Y_{i}=X_{i} / / G$. Therefore, Lemma 4.2.9 and Corollary 4.2.11 extend to the relative situation.

Theorem 5.1.2. Assume that a scheme $X$ is provided with a relatively affine action of a diagonalizable group $G$.

(i) The morphism $X \rightarrow Y=X / / G$ is submersive and $Y$ is the universal categorical quotient of the action.

(ii) For each $y \in Y$, the fiber $X_{y}$ contains a single orbit $O$ which is closed in $X_{y}$, and this orbit belongs to the closure of any other orbit contained in $X_{y}$.

From here on, we will consider only relatively affine actions.

Remark 5.1.3. The notion of a relatively affine action is not as meaningful for non-reductive groups because it does depend on $Z$. For instance, in the situation of Example 4.2.12, we can take $Z$ to be either $\operatorname{Spec}(k)$ or $\mathbb{P}_{k}^{1}$. For both choices, the relative quotient coincides with $Z$.

5.1.4 Strongly equivariant open subschemes. Assume that $X$ is provided with a relatively affine action of $G=\mathbf{D}_{L}$. An open subscheme $U \hookrightarrow X$ is called strongly equivariant if it is the preimage of an open subscheme $V \hookrightarrow Y / / G$. Note that $V=U / / G$. We used a covering of $X$ by strongly equivariant schemes to prove Theorem 5.1.2. In general, one can use strongly equivariant open subschemes to work locally on $X / / G$ without describing the quotients explicitly.

Lemma 5.1.5. Assume that a diagonalizable group $G=\mathbf{D}_{L}$ acts on a scheme $X$ and that we have a covering of $X$ by open equivariant subschemes $X_{i}$. If the action on each $X_{i}$ is relatively 


\section{LUNA'S FUNDAMENTAL LEMMA FOR DIAGONALIZABLE GROUPS}

affine and each intersection $X_{i j}=X_{i} \cap X_{j}$ is strongly equivariant in both $X_{i}$ and $X_{j}$, then the action on $X$ is relatively affine and each $X_{i}$ is strongly equivariant in $X$.

Proof. By definition, the schemes $Y_{i}=X_{i} / / G$ glue along their open subschemes $Y_{i j}=X_{i j} / / G$ to form a scheme $Y$. Hence the quotient morphisms $X_{i} \rightarrow Y_{i}$ glue to an affine quotient morphism $X \rightarrow Y$.

Example 5.1.6. If $X=\operatorname{Spec}(A)$, where $A$ is $L$-graded, and $f \in A$ is a homogeneous element, then $U=\operatorname{Spec}\left(A_{f}\right)$ is equivariant but usually not strongly equivariant. However, it is strongly equivariant if $f$ is of degree zero. In particular, an important particular case of Lemma 5.1.5 is when the $X_{i}$ are affine and each $X_{i j}$ is a localization of both $X_{i}$ and $X_{j}$ at elements of degree zero.

5.1.7 Geometric quotients. If the action of $G$ on $X$ is relatively affine and each fiber of the quotient morphism $p: X \rightarrow X / / G$ consists of a single orbit, then we say that the quotient is geometric and use the notation $X / G$ instead of $X / / G$. This matches the terminology, but not the notation, of geometric invariant theory (GIT); see [MFK94, Definition 0.6].

5.1.8 Special orbits. If an orbit of a $G$-action on $X$ is closed in the fiber of $X \rightarrow X / / G$, then we say that the orbit is special. Obviously, such an orbit is a scheme.

5.1.9 Local actions. We say that a relatively affine action of $G$ on a scheme $X$ is local if $X$ is quasi-compact and contains a single closed orbit.

Lemma 5.1.10. Assume that we have a relatively affine action of $G=\mathbf{D}_{L}$ on a scheme $X$. Then the following conditions are equivalent:

(i) The action is local.

(ii) The scheme $X$ is affine, say $X=\operatorname{Spec}(A)$, and the $L$-graded $\operatorname{ring} A$ is $L$-local.

(iii) The quotient $Y=X / / G$ is local.

Proof. A scheme is local if and only if it is quasi-compact and contains a single closed point. Therefore, conditions (i) and (iii) are equivalent. The equivalence of conditions (ii) and (iii) was proved in Lemma 4.4.5.

5.1.11 Localization along a special orbit. Assume that $O$ is a special orbit of a relatively affine action on $X$ and $y$ is its image in $Y$. Consider the localization $Y_{y}=\operatorname{Spec}\left(\mathcal{O}_{Y, y}\right)$, and set $X_{O}=X \times_{Y} Y_{y}$. We call $X_{O}$ the equivariant localization of $X$ along $O$. Note that $X_{O} / / G=Y_{y}$ by the universality of the quotient; in particular, $G$ acts locally on $X_{O}$.

Remark 5.1.12. (i) Set-theoretically, $X_{O}$ consists of all orbits whose closure contains $O$. So, even if $O=\{x\}$ is a closed point, it typically happens that $X_{O}$ is larger than the localization of $X$ at $x$. Equivariant localization of a scheme $X=\operatorname{Spec}(A)$ corresponds to homogeneous localization of $A$ in the sense of Example 4.4.6.

(ii) Even if $O$ is only locally closed in the fiber of $X \rightarrow X / / G$, one can define an equivariant localization $X_{O} \hookrightarrow X$ whose only closed orbit is $O$. We will not use this construction. If $O$ is not special, then the localization morphism $X_{O} \hookrightarrow X$ is not inert (see Section 5.5 below), and the morphism $X_{O} / / G \rightarrow Y$ can be bad (for example non-flat). 


\section{Abramovich and M. Temkin}

5.1.13 The schemes of fixed points. If $X$ is acted on by $G$, then the scheme of fixed points of $X$ is the maximal closed subscheme $X^{G}$ of $X$ such that $X^{G}$ is equivariant and the action of $G$ on it is trivial. In other words, it is the maximal closed subscheme over which the inclusion $I_{X} \hookrightarrow G \times X$ becomes an isomorphism. For diagonalizable groups, the existence and functoriality of fixed-point schemes is guaranteed by [SGA3-2, Exemple VIII.6.5(a)], where one sets $Y=G$ and $Z=X \times X$, and $Z^{\prime} \subset Z$ is the diagonal. If $X=\operatorname{Spec}(A)$ is affine, then, as we noted in Section 4.1.2, we have $X^{G}=\operatorname{Spec}\left(A_{G}\right)$.

5.1.14 Inertia stratification. If $G$ is diagonalizable and $G^{\prime} \subseteq G$ is a subgroup, then

$$
X\left(G^{\prime}\right):=X^{G^{\prime}} \backslash \cup_{G^{\prime \prime} \supsetneq G^{\prime}} X^{G^{\prime \prime}}
$$

is the maximal $G$-equivariant subscheme $Y$ with constant inertia equal to $G^{\prime}$ (that is, such that $\left.I_{Y}=G^{\prime} \times Y\right)$. The family of subschemes $\left\{X\left(G^{\prime}\right)\right\}_{G^{\prime} \subseteq G}$ provides a $G$-equivariant stratification of $X$ that we call the inertia stratification. Set-theoretically, this is the stratification of $X$ by the stabilizers of points.

5.1.15 Regularity. The fixed-points functor preserves regularity; see [Fog73, Corollary of Theorem 5.4]. We provide a simple proof in our situation.

Proposition 5.1.16. Assume that a diagonalizable group $G$ acts on a regular scheme $X$. Then the scheme of fixed points $X^{G}$ is regular. In particular, the strata of the inertia stratification of $X$ are regular.

Proof. The claim is local at a point $x \in X^{G} \hookrightarrow X$. Since $x$ is $G$-invariant, we can replace $X$ with the equivariant localization along $x$. Then $X$ is the spectrum of a strictly $L$-local ring, and it remains to use Lemma 4.5.4.

5.1.17 The case of $\mathbb{G}_{m}$. We discuss a construction which is specific to $G=\mathbb{G}_{m}=\mathbf{D}_{\mathbb{Z}}$ and will be used in [AT16]; we indicate the general case in Remark 5.1.19 below. Assume that $X$ is provided with a relatively affine action of $G$. Following [Wło00], we define $X_{+}$and $X_{-}$to be the open subschemes obtained by removing all orbits that have a limit at $+\infty$ and $-\infty$, respectively. The construction of $X_{ \pm}$is local on $X / / G$ and if the latter is affine, say $X=\operatorname{Spec} A$ and $X / / G=\operatorname{Spec} A_{0}$, then $X_{+}=X \backslash V\left(A_{-}\right)$with $A_{-}=\oplus_{n<0} A_{n}$. Similarly, $X_{-}=X \backslash V\left(A_{+}\right)$ for $A_{+}=\oplus_{n>0} A_{n}$.

Lemma 5.1.18. Assume that $G=\mathbb{G}_{m}$ acts in a relatively affine manner on a scheme $X$.

(i) The group $G$ acts in a relatively affine manner on $X_{+}$and $X_{-}$.

(ii) If $X=\operatorname{Spec}(A)$ is affine, then the schemes $X_{f}=\operatorname{Spec}\left(A\left[f^{-1}\right]\right)$ with homogeneous $f \in A_{-}$ (respectively, $f \in A_{+}$) form an open strongly equivariant covering of $X_{+}$(respectively, $X_{-}$).

Proof. If $f \in A_{-}$, then $V\left(A_{-}\right) \subseteq V(f)$, and hence $X_{f}$ is an equivariant open subscheme of $X_{+}$. By definition, $X_{+}=\cup_{f \in A_{m}, m<0} X_{f}$. By Lemma 5.1.5, it suffices to check that $X_{f g}$ is strongly equivariant in $X_{f}$ for $f \in A_{n}$ and $g \in A_{m}$ with $m, n<0$. It remains to notice that $X_{f g}$ can be described as the localization of $X_{f}$ obtained by inverting the degree zero element $f^{-n} g^{m} \in A\left[f^{-1}\right]$; see Remark 5.1.6.

Remark 5.1.19 (see [Tha96]). For arbitrary $G=\mathbf{D}_{L}$ and affine $X=\operatorname{Spec} A$, one defines $X / / m G=$ $\operatorname{Proj} A[z]^{G}$ with $G$ acting on $z$ via $-m \in L$. Then there is a locally finite decomposition $\Sigma=\coprod \sigma_{i}^{\circ}$ of the monoid $\Sigma \subset L$ of characters figuring in $A$ into relative interiors of polyhedral cones, and 


\section{LUNA'S FUNDAMENTAL LEMMA FOR DIAGONALIZABLE GROUPS}

$X / / m G=X / / \sigma^{\circ} G$ is constant on such interiors of cones. The replacement of $X_{ \pm}$is $X^{s s}(m)$, the semistable locus of $X$ with respect to the linearization provided by the character $m$.

\subsection{Basic properties of the quotient functor}

In this section, we study the quotient map $X \rightarrow X / / G$ and properties of schemes and morphisms preserved by the quotient functor. The most subtle result is that the quotient map is finite when $X$ is noetherian.

\subsubsection{Quotients of schemes.}

Theorem 5.2.2. Assume that a diagonalizable group $G=\mathbf{D}_{L}$ acts trivially on a scheme $S$ and that an $S$-scheme $X$ is provided with a relatively affine action of $G$.

(i) Assume that $X$ satisfies one of the following properties: (a) reduced, (b) integral, (c) normal with finitely many connected components, (d) locally of finite type over $S$, (e) of finite type over $S$, (f) quasi-compact over $S$, (g) locally noetherian, (h) noetherian. Then $X / / G$ satisfies the same property.

(ii) If $X$ is locally noetherian, then the quotient morphism $X \rightarrow X / / G$ is of finite type.

Proof. Note that $X \rightarrow S$ factors through $Y=X / / G$ because the latter is the categorical quotient by Theorem 5.1.2. Claim (f) is obvious since $X \rightarrow Y$ is onto. Furthermore, a morphism is of finite type if and only if it is quasi-compact and locally of finite type; hence claim (e) follows from claims (d) and (f). It remains to prove all assertions except claims (e) and (f). As shown in [MFK94, Chapter 0, Section 2], claims (a), (b), and (c) hold for any categorical quotient; hence they are implied by Theorem 5.1.2(i). Note that the assertion of claim (d) is local on $S$; hence we can assume that $S$ is affine. Furthermore, all assertions of the theorem we are dealing with are local on $Y$, so we can assume that $Y$ is affine. In this case, claim (d) was proven in Lemma 4.2.4, and claims (g) and (h) and part (ii) were proven in Proposition 4.3.1.

\subsubsection{Quotients of morphisms. We start with the following corollary of Theorem 5.2.2.}

Corollary 5.2.4. Assume that locally noetherian schemes $X$ and $X^{\prime}$ are provided with relatively affine actions of a diagonalizable group $G$ and that $f: X^{\prime} \rightarrow X$ is a $G$-equivariant morphism. If $f$ is of finite type, then the quotient morphism $f / / G$ is of finite type.

Proof. The morphism $X \rightarrow X / / G$ is of finite type by Theorem 5.2.2(ii). Hence the composition $X^{\prime} \rightarrow X / / G$ is of finite type, and then $X^{\prime} / / G \rightarrow X / / G$ is also of finite type by Theorem 5.2.2(i)(d).

Proposition 5.2.5. Let $G$ be a diagonalizable group and $f: X^{\prime} \rightarrow X$ a $G$-equivariant morphism such that the actions on $X$ and $X^{\prime}$ are relatively affine. Then:

(i) If $f$ satisfies one of the following properties: (a) affine, (b) integral, (c) a closed embedding, then $f / / G$ satisfies the same property.

(ii) If $X$ is locally noetherian and $f$ is finite, then $f / / G$ is finite.

Proof. The claim is local on $Y=X / / G$; hence we can assume that $X=\operatorname{Spec}(A)$ and $Y=$ $\operatorname{Spec}\left(A_{0}\right)$ are affine. Since $f$ is affine in each case, $X^{\prime}=\operatorname{Spec}\left(A^{\prime}\right)$ is affine, and so $Y^{\prime}=\operatorname{Spec}\left(A_{0}^{\prime}\right)$ is affine. This proves claim (a). If $A \rightarrow A^{\prime}$ is onto, then $A_{0} \rightarrow A_{0}^{\prime}$ is onto and we obtain claim (c).

If $A \rightarrow A^{\prime}$ is integral, then any $x \in A^{\prime}$ satisfies an integral equation $x^{n}+\sum_{i=0}^{n-1} a_{i} x^{i}$ with coefficients in $A$. If $x \in A_{0}^{\prime}$, then replacing each $a_{i}$ with its component of degree zero, we obtain 


\section{Abramovich and M. Temkin}

an integral equation for $x$ with coefficients in $A_{0}$. Thus, $A_{0}^{\prime}$ is integral over $A_{0}$ and we obtain claim (b). Finally, part (ii) follows from claim (b) and Corollary 5.2.4.

5.2.6 Some bad examples. Basic properties of $G$-morphisms, including smoothness and separatedness, are not preserved by the quotient functor. Here are some classical bad examples.

EXAmPle 5.2.7. (i) Let $k$ be a field with char $k \neq 2$, and let $X=\operatorname{Spec}(k[x, y])$ be an affine plane with $\mu_{2}$ acting by changing the sign of $x$ and $y$. Then $Y=X / / \mu_{2}$ is the quotient singularity $Y=\operatorname{Spec}\left(k\left[x^{2}, x y, y^{2}\right]\right)$, and the quotient morphism $X \rightarrow Y$ is not even flat. On the other hand, set $X^{\prime}=X \times \mu_{2}$ with $\mu_{2}$ acting diagonally. The projection $X^{\prime} \rightarrow X$ is a split étale covering which is not inert above the origin $O \in X$ because $O$ is fixed by $\mu_{2}$ and the action of $\mu_{2}$ on $X^{\prime}$ is free. Moreover, $Y^{\prime}=X^{\prime} / / \mu_{2} \stackrel{\sim}{\rightarrow} X$, so the quotient map $f / / \mu_{2}$ is the projection $X \rightarrow Y$. Although $f$ is split étale, its quotient is not even flat.

(i)' Similarly, it is easy to construct a $\mathbb{G}_{m}$-action on $X=\mathbb{A}_{k}^{3}$ such that the quotient $Y=X / / \mathbb{G}_{m}$ is not smooth (for example, if $X=\operatorname{Spec}(A)$ for $A=k[x, y, z]$ with $x, y \in A_{1}$ and $z \in A_{-2}$, then $Y=\operatorname{Spec}\left(k\left[x^{2} z, x y z, y^{2} z\right]\right)$ is an orbifold). Then, automatically, the morphism $X \rightarrow Y$ is not flat, and setting $X^{\prime}=X \times \mathbb{G}_{m}$, we obtain another bad example, where $X^{\prime} \rightarrow X$ is smooth but the morphism between $X^{\prime} / / \mathbb{G}_{m}=X$ and $X / / \mathbb{G}_{m}$ is not flat.

(ii) Let $k$ be a field and $G=\mathbb{G}_{m}=\mathbf{D}_{\mathbb{Z}}$. Consider the $\mathbb{Z}$-graded $k$-algebra $A=k[x, y, z]$ with $x \in$ $A_{1}$ and $y, z \in A_{-1}$, and set $X=\operatorname{Spec}(A)=\mathbb{A}_{k}^{3}$. Then $Y=X / / G$ equals $\operatorname{Spec}(k[x z, x y])=\mathbb{A}_{k}^{2}$, and the orbits over the origin are the origin, the punctured $(x)$-axis, and all punctured lines through the origin in the $(y z)$-plane. Consider the equivariant subspace $X^{\prime}=\operatorname{Spec}\left(k\left[x, y, z^{ \pm 1}\right]\right)$. The open embedding $X^{\prime} \hookrightarrow X$ preserves stabilizers but takes some closed orbits to non-closed orbits. For example, the punctured $(z)$-axis is closed in $X^{\prime}$. The quotient $Y^{\prime}=X^{\prime} / / G=\operatorname{Spec}(k[x z, y / z])$ is not flat over $Y$. In fact, it is an affine chart of the blowing up of $Y$ at the origin, and the quotient map $X^{\prime} \rightarrow Y^{\prime}$ separates all orbits of $X$ sitting over the origin of $Y$ and contained in $X^{\prime}$.

(ii)' Analogous examples related to cobordisms will play a crucial role in the proof of the factorization theorem: $\left(B_{a}\right)_{+} \hookrightarrow B_{a}$ is an open embedding preserving the stabilizers (see [AT16, $\S 3.4])$, but $\left(B_{a}\right)+/ / G \rightarrow B_{a} / / G$ is usually a non-trivial modification.

(iii) Let $G=\mathbb{G}_{m}$ act on $T=\operatorname{Spec}(k[x, y])$ as $x \mapsto t x$ and $y \mapsto t^{-1} y$, and let $X^{\prime}$ be obtained from $T$ by removing the origin. Then $X^{\prime} / / G$ is an affine line with doubled origin. In particular, the morphism $f: X^{\prime} \rightarrow \operatorname{Spec}(k)$ is separated, but its quotient is not.

\subsection{Strongly equivariant morphisms}

The situation improves drastically if one considers quotients of a more restrictive class of strongly equivariant morphisms.

5.3.1 The definition. We say that a $G$-morphism $f: X^{\prime} \rightarrow X$ is strongly equivariant if the actions are relatively affine and $f$ is the base change of its quotient $f / / G$, that is, the morphism $\phi: X^{\prime} \rightarrow X \times_{Y} Y^{\prime}$ is an isomorphism, where $Y=X / / G$ and $Y^{\prime}=X^{\prime} / / G$. Furthermore, we say that $f$ is strongly equivariant over a point $y^{\prime} \in Y^{\prime}$ if $\phi$ is an isomorphism over $y^{\prime}$ in the sense that

$$
\phi_{y^{\prime}}: X^{\prime} \times_{Y^{\prime}} \operatorname{Spec}\left(\mathcal{O}_{Y^{\prime}, y^{\prime}}\right) \rightarrow X \times_{Y} \operatorname{Spec}\left(\mathcal{O}_{Y^{\prime}, y^{\prime}}\right)
$$

is an isomorphism. Note that $f$ is strongly equivariant if and only if it is strongly equivariant over all points of $Y^{\prime}$, and if $Y^{\prime}$ is quasi-compact, then it suffices to consider only closed points. 


\section{LUNA'S FUNDAMENTAL LEMMA FOR DIAGONALIZABLE GROUPS}

Lemma 5.3.2. Let $G=\mathbf{D}_{L}$ be a diagonalizable group.

(i) The composition of strongly $G$-equivariant morphisms is strongly $G$-equivariant.

(ii) If $Y \rightarrow X$ is a strongly $G$-equivariant morphism and $g: Z \rightarrow Y$ is a $G$-equivariant morphism such that the composition is strongly $G$-equivariant, then $g$ is strongly $G$-equivariant.

(iii) If $Y \rightarrow X$ is strongly $G$-equivariant and $Z \rightarrow X$ is $G$-equivariant, then the base change $Y \times_{X} Z \rightarrow Z$ is strongly $G$-equivariant.

(iv) If $f: Y \rightarrow X$ is strongly equivariant, then the diagonal $\Delta_{f}: Y \rightarrow Y \times_{X} Y$ is strongly equivariant and $\Delta_{f} / / G$ is the diagonal of $f / / G$.

Proof. The proof of parts (i), (ii), and (iv) is a simple diagram chase of the relevant cartesian squares, so we omit it. Let us prove part (iii). The claim is local on $X / / G, Y / / G$, and $Z / / G$; hence we can assume $X=\operatorname{Spec}(A), Y=\operatorname{Spec}(B)$, and $Z=\operatorname{Spec}\left(A^{\prime}\right)$ for $L$-graded $\operatorname{rings} A, B, A^{\prime}$. We are given that $B=A \otimes_{A_{0}} B_{0}$, and we should prove that $B^{\prime}=A^{\prime} \otimes_{A} B$ satisfies $B^{\prime}=A^{\prime} \otimes_{A_{0}^{\prime}} B_{0}^{\prime}$. Clearly, $B^{\prime}=A^{\prime} \otimes_{A_{0}} B_{0}$ and so $B_{n}^{\prime}=A_{n}^{\prime} \otimes_{A_{0}} B_{0}$ for any $n \in L$. Therefore, $B_{n}^{\prime}=A_{n}^{\prime} \otimes_{A_{0}^{\prime}} A_{0}^{\prime} \otimes_{A_{0}} B_{0}=$ $A_{n}^{\prime} \otimes_{A_{0}^{\prime}} B_{0}^{\prime}$, as required.

5.3.3 Strongly satisfied properties. Let $P$ be a property of morphisms preserved by base changes. As in Section 4.6, we say that an equivariant morphism $f: X^{\prime} \rightarrow X$ strongly satisfies $P$ if it is strongly equivariant and the quotient $f / / G$ satisfies $P$. In particular, $f$ itself satisfies $P$.

Remark 5.3.4. (i) In the case of strongly étale morphisms, we recover the definition from Appendix 1.D of [MFK94]. Such morphisms play an important role in [AKMW02], and we will use strongly regular morphisms in [AT16] for analogous purposes.

(ii) Let $U$ be an open subscheme of $X$. The morphism $U \hookrightarrow X$ is a strongly open immersion if and only if $U$ is a strongly equivariant open subscheme.

(iii) For various properties $P$, it is true that a morphism strongly satisfies $P$ if and only if it satisfies $P$ and is strongly equivariant. In fact, one should only check that if $f$ satisfies $P$ and is strongly equivariant, then $f / / G$ satisfies $P$. However, such descent results may be difficult to prove because the morphisms $X \rightarrow X / / G$ are not always flat; in particular, flat descent is unapplicable. For the strong étale property, such a descent claim is a part of Luna's fundamental lemma.

5.3.5 Some descent results. Here is a (rather incomplete) list of properties for which the descent is easy.

Proposition 5.3.6. Let $G$ be a diagonalizable group and $f: X^{\prime} \rightarrow X$ a $G$-equivariant morphism of schemes with relatively affine $G$-actions. Then:

(i) Let $P$ be any of the following properties: (a) has finite fibers, (b) a monomorphism, (c) separated, (d) universally closed. Then $f$ strongly satisfies $P$ if and only if it satisfies $P$ and is strongly equivariant.

(ii) Assume that $X$ is locally noetherian, and let $P$ be one of the following properties: (e) quasifinite, (f) proper. Then $f$ strongly satisfies $P$ if and only if it satisfies $P$ and is strongly equivariant.

Proof. In all claims, we assume that $f$ is strongly equivariant and satisfies $P$, and we should prove that $g=f / / G$ satisfies $P$. Assertion (a) follows from the surjectivity of $X \rightarrow X / / G$. Note that a morphism is a monomorphism if and only if its diagonal is an isomorphism. Hence 


\section{Abramovich and M. Temkin}

assertion (b) follows from Lemma 5.3.2(iv). Similarly, assertion (c) follows from Lemma 5.3.2(iv) and Proposition 5.2.5(i)(c).

(d) First, assume only that $f$ is closed. Then for any closed set $T \subset X^{\prime} / / G$, the preimage of $g(T)$ in $X$ is closed. Since the quotient morphism $X \rightarrow X / / G$ is submersive, $g(T)$ is closed. Thus $g$ is closed. Part (ii) follows from this and the fact that the base change of $f$ by a strongly equivariant morphism is strongly equivariant by Lemma 5.3.2(iii).

Finally, assertion (e) follows from assertion (a) and Corollary 5.2.4, and assertion (f) follows from assertion (d) and Corollary 5.2.4.

5.3.7 The case of $\mathbb{G}_{m}$. Assume that $X$ and $Y$ are provided with a relatively affine action of $G=\mathbb{G}_{m}$. We refer to Section 5.1.17 for the definitions of $X_{ \pm}$and $Y_{ \pm}$.

Lemma 5.3.8. If $f: X \rightarrow Y$ is strongly equivariant, then $X_{ \pm}=Y_{ \pm} \times_{Y} X$.

Proof. The claim is local on $Y / / G$ and $X / / G$, so we can assume $Y=\operatorname{Spec} B, Y / / G=\operatorname{Spec} B_{0}$, $X=\operatorname{Spec} A$, and $X / / G=\operatorname{Spec} A_{0}$. By strong equivariance, $A=B \otimes_{B_{0}} A_{0}$. Hence $A_{n}=B_{n} \otimes_{B_{0}} A_{0}$ for any $n$, and we obtain equalities of ideals $B_{-} A=A_{-}$and $B_{+} A=A_{+}$.

Remark 5.3.9. For a general $G=\mathbf{D}_{L}$, we similarly have $X^{s s}(m)=\left(Y^{s s}(m)\right) \times_{Y} X$.

\subsection{Free actions}

In Section 4.4, we studied the quotients when the stabilizers are maximal. This section is devoted to the other extreme case when the stabilizers are trivial. We will show in Corollary 5.4.5 that any quotient of a relatively affine action of $G=\mathbf{D}_{L}$ can be described in terms of these two cases.

5.4.1 Definitions. We say that an action of $G$ on $X$ is regular or split free if there is an equivariant isomorphism $X \stackrel{\sim}{\rightarrow} G \times Y$, where $Y=X / / G$. If such an isomorphism only exists locally on $Y$ for a topology $\tau$ (for example flat, étale, or Zariski), then we say that the action is $\tau$-split free. Finally, if there exist a $\tau$-open morphism $g: Y^{\prime} \rightarrow Y$ and an equivariant isomorphism $X \times_{Y} Y^{\prime} \stackrel{\sim}{\rightarrow} G \times Y^{\prime}$, then for any point $y \in g\left(Y^{\prime}\right)$, we say that the action is $\tau$-split free over $y$.

Remark 5.4.2. Recall that an action of $G$ on $X$ is semi-regular or free if the morphism $G \times$ $X \rightarrow X \times X$ is a closed embedding, and this condition is equivalent to the condition that $\psi: G \times X \rightarrow X \times_{Y} X$ is an isomorphism; in other words, $X$ is a pseudo $G$-torsor over $Y$; see [SP17, tag/0498]. This pseudo-torsor is a $\tau$-torsor of $G$ if and only if the action is $\tau$-split free. In fact, any semi-regular action has free orbits; hence it follows from Lemma 5.4.4 below that it is flat-split free over the quotient $Y$. In particular, we will not distinguish free and flat-split free actions.

5.4.3 A criterion for splitting. The flat-split freeness of an action can be tested very easily: the stabilizers of the points should be trivial.

Lemma 5.4.4. Given a relatively affine action of a diagonalizable group $G=\mathbf{D}_{L}$ on a scheme $X$, let $f: X \rightarrow Y=X / / G$ be the quotient morphism and $y \in Y$ a point. Then the following conditions are equivalent:

(i) The action is flat-split free over $y$.

(ii) The scheme-theoretic fiber $X_{y}=y \times_{Y} X$ coincides with a single free orbit $O=\operatorname{Spec}(k[L])$.

(iii) The set-theoretic fiber $f^{-1}(y)$ is a single free orbit. 


\section{LUNA'S FUNDAMENTAL LEMMA FOR DIAGONALIZABLE GROUPS}

(iv) All points of the fiber $f^{-1}(y)$ have trivial stabilizer.

Moreover, if the degree of the torsion of $L$ is invertible on $Y$ (respectively, $L$ is torsion free), then one may replace the flat topology in part (i) with the étale (respectively, Zariski) topology.

Proof. The implications (i) $\Longrightarrow$ (ii) $\Longrightarrow$ (iii) $\Longrightarrow$ (iv) are obvious. In the opposite direction, if part (iv) holds, then all orbits in $f^{-1}(y)$ are free and closed, hence the fiber is a single orbit by Theorem 5.1.2(ii), and we obtain part (iii).

If part (iii) holds, then $X_{y}=\operatorname{Spec}(A)$ with $A$ such that $A_{0}=k$ and $k[L]$ is the reduction of $A$. This implies that each $A_{n}$ contains a unit and hence is an invertible $k$-module. Therefore, the reduction $A \rightarrow k[L]$ is an isomorphism, and we obtain part (ii).

Finally, assume $X_{y}=\operatorname{Spec}(k[L])$. Let $L=\oplus_{i=1}^{n} L_{i}$ be a decomposition of $L$ into cyclic groups, and choose a generator $m_{i}$ of $L_{i}$. Shrinking $Y$ around $y$, we can assume $X=\operatorname{Spec}(A)$ and $Y=\operatorname{Spec}\left(A_{0}\right)$, and that each $m_{i} \in k[L]$ lifts to a unit $u_{i} \in A_{m_{i}}$ with respect to the homomorphism $A \rightarrow k[L]$. For each $m_{i}$ of a finite order $d_{i}$, we have $u_{i}^{d_{i}}=a_{i} \in A_{0}$. Obviously, $X$ is a principal $G$-torsor over $Y$ which is trivialized by adjoining the roots $a_{i}^{1 / d_{i}}$ to $A_{0}$. The latter defines a flat covering of $Y$, which is étale (respectively, an isomorphism) if the $d_{i}$ are invertible on $Y$ (respectively, $L$ is torsion free).

The following corollary shows that, locally, one can construct quotients in two steps: first by dividing by the stabilizer and then by dividing by a free action.

Corollary 5.4.5. Assume that a scheme $X$ is provided with a relatively affine action of a diagonalizable group $G=\mathbf{D}_{L}$, that $y$ is a point of $Y=X / / G$, that $G^{\prime}$ is the stabilizer of the closed orbit of the fiber $X_{y}$, that $Z=X / / G^{\prime}$, and that $G^{\prime \prime}=G / G^{\prime}$. Then $Z / / G^{\prime \prime}=Y$ and the $G^{\prime \prime}$-action on $Z$ is flat-split free over $y$. Moreover, if the torsion of $L$ is invertible on $Y$ (respectively, $L$ is torsion free), then the action is étale-split (respectively, Zariski-split) free over $y$.

Proof. By universality, quotients are compatible with taking fibers. So, in view of Lemma 5.4.4, it suffices to show that $X_{y} / / G^{\prime}$ is a single free $G^{\prime \prime}$-orbit. Note that $X_{y}=\operatorname{Spec}(A)$, where $(A, m)$ is an $L$-local ring with $A_{0}=k$. Let $0 \rightarrow L^{\prime \prime} \rightarrow L \rightarrow L^{\prime} \rightarrow 0$ be the exact sequence corresponding to $1 \rightarrow G^{\prime} \rightarrow G \rightarrow G^{\prime \prime} \rightarrow 1$. Since $A / m=k\left[L^{\prime \prime}\right]$, each $A_{n}$ with $n \in L^{\prime \prime}$ contains a unit, and we obtain $A^{L^{\prime}}=\oplus_{n \in L^{\prime \prime}} k=k\left[L^{\prime \prime}\right]$. Thus, $X_{y} / / G^{\prime}=\operatorname{Spec}\left(k\left[L^{\prime \prime}\right]\right)$, and we are done.

5.4.6 Descent of regularity. We show below that dividing by a free action is a nice functor that preserves various properties of equivariant morphisms. In fact, the following lemma reduces this to the usual flat descent.

Lemma 5.4.7. Assume that a diagonalizable group $G$ acts on a scheme $X$ and the action is flat-split free over a point $y \in Y=X / / G$. Then the quotient morphism $f: X \rightarrow Y$ is flat along the fiber $f^{-1}(y)$. In particular, if $X$ is regular at a point $x \in f^{-1}(y)$, then $Y$ is regular at $y$.

Proof. By definition, there exist a flat morphism $Y^{\prime} \rightarrow Y$ whose image contains $y$ and an isomorphism $X \times_{Y} Y^{\prime} \stackrel{\sim}{\rightarrow} G \times Y^{\prime}$. Then the flat base change $X \times_{Y} Y^{\prime} \rightarrow Y^{\prime}$ of $f$ is flat, and hence $f$ is flat over $y$. If $X$ is regular at a point $x$, then $Y$ is regular at $y$ by [SP17, tag/00OF].

Corollary 5.4.8. Assume that schemes $X$ and $X^{\prime}$ are provided with relatively affine actions of a diagonalizable group $G=\mathbf{D}_{L}$ and that $f: X^{\prime} \rightarrow X$ is a $G$-equivariant morphism with quotient $g=f / / G: Y^{\prime} \rightarrow Y$. Suppose that $y^{\prime} \in Y^{\prime}$ is a point such that the actions on $X^{\prime}$ and $X$ are flat-split free over $y^{\prime}$ and $g\left(y^{\prime}\right)$, respectively. Then $f$ is strongly equivariant over $y^{\prime}$. In particular, if $f$ is regular or lci at a point $x^{\prime} \in X_{y^{\prime}}^{\prime}$, then $g$ is regular or lci at $y^{\prime}$, respectively. 


\section{Abramovich and M. Temkin}

Proof. The statement is local at $y^{\prime}$ and $y$. So, we can assume that $Y$ and $Y^{\prime}$ are local, and the claim reduces to showing that $\phi: X^{\prime} \rightarrow X \times_{Y} Y^{\prime}$ is an isomorphism. By flat descent, this can be checked flat-locally on $Y$, so by Lemma 5.4 .7 we can assume $X \stackrel{\sim}{\rightarrow} G \times Y$. By the same lemma, there exists a flat covering $Y^{\prime \prime} \rightarrow Y^{\prime}$ such that $X^{\prime} \times_{Y^{\prime}} Y^{\prime \prime} \stackrel{\sim}{\rightarrow} G \times Y^{\prime \prime}$. The base change of $\phi$ with respect to the morphism $Y^{\prime \prime} \rightarrow Y^{\prime}$ is the isomorphism $X^{\prime} \times_{Y^{\prime}} Y^{\prime \prime} \stackrel{\sim}{\rightarrow} G \times Y^{\prime \prime} \stackrel{\sim}{\rightarrow} X \times_{Y} Y^{\prime \prime}$. Thus, $\phi$ is an isomorphism by flat descent.

Suppose that $f$ is regular. To check that $g: Y^{\prime} \rightarrow Y$ is regular or lci, it suffices to check that its pullback by a flat surjective morphism is regular or lci, respectively; but $X \rightarrow Y$ is flat and surjective by Lemma 5.4.7, and we have shown that the pullback is $f: X^{\prime} \rightarrow X$.

\subsection{Inert morphisms}

The main disadvantage in the definition of strong equivariance (Section 5.3.1) is that it involves the quotient morphism. It is desirable to have an explicit criterion of strong equivariance in terms of the morphism itself. This leads to the notion of inert morphisms.

5.5.1 Inertia-preserving morphisms. Recall that a $G$-equivariant morphism $f: X^{\prime} \rightarrow X$ is called fixed-point reflecting if for any $x^{\prime} \in X^{\prime}$ and $x=f\left(x^{\prime}\right)$, the inclusion of the stabilizers $G_{x^{\prime}} \hookrightarrow G_{x}$ is an equality (see, for example, [Knu71, IV.1.8]). We propose the following schemetheoretic strengthening of this notion: a $G$-equivariant morphism $f: X^{\prime} \rightarrow X$ is inertia preserving if the closed immersion $I_{X^{\prime}} \hookrightarrow I_{X} \times_{X} X^{\prime}$ is an equality.

Lemma 5.5.2. Assume that locally noetherian schemes $X$ and $X^{\prime}$ are provided with relatively affine actions of a diagonalizable group $G=\mathbf{D}_{L}$ and that $f: X^{\prime} \rightarrow X$ is a $G$-equivariant morphism. Consider the following conditions:

(i) The morphism $f$ is inertia preserving.

(ii) For each subgroup $H \subseteq G$, the closed immersion $X^{\prime H} \hookrightarrow X^{H} \times_{X} X^{\prime}$ is an equality.

(iii) For each point $x \in X$, the stabilizer $G_{x}$ acts trivially on the fiber $X^{\prime} \times_{X} x$.

(iv) The morphism $f$ is fixed-point reflecting and for any point $x^{\prime} \in X^{\prime}$ with $x=f\left(x^{\prime}\right)$, the action of $G_{x}$ on $\Omega_{X^{\prime} / X} \otimes k\left(x^{\prime}\right)$ is trivial.

(v) The morphism $f$ is fixed-point reflecting.

Then conditions (ii), (iii), (iv) are equivalent, each condition is implied by condition (i), and all conditions imply condition (v). In addition, if $f$ has reduced fibers, then condition (v) implies the conditions (ii)-(iv).

Proof. The implications (i) $\Longrightarrow$ (ii) $\Longrightarrow$ (iii) and (iv) $\Longrightarrow(\mathrm{v})$ are obvious, and the equivalence (iii) $\Longleftrightarrow$ (iv) follows from Lemma 4.6.4 since $\Omega_{X^{\prime} / X} \otimes k\left(x^{\prime}\right)=\Omega_{Z_{x} / x} \otimes k\left(x^{\prime}\right)$ for the fiber $Z_{x}=$ $X^{\prime} \times{ }_{X} x$.

We show (iii) $\Longrightarrow$ (ii). Assume that (ii) fails, that is, there exists a subgroup $H=\mathbf{D}_{L^{\prime}} \subseteq G$ which acts non-trivially on $X^{H} \times_{X} X^{\prime}$. It suffices to prove that in this case $H$ acts non-trivially already on the fiber $Z_{x}$ over a point $x \in X^{H}$. Choose an open affine subscheme $\operatorname{Spec}(A) \hookrightarrow X^{H}$ such that the action of $H$ on $\operatorname{Spec}(B)=\operatorname{Spec}(A) \times_{X} X^{\prime}$ is non-trivial. The morphism $\operatorname{Spec}(B) \rightarrow$ $\operatorname{Spec}(A)$ corresponds to an $L^{\prime}$-homogeneous homomorphism $A \rightarrow B$ such that $A=A_{0}$ and $B_{l} \neq 0$ for some $l \neq 0$. Note that $B_{l}$ is finitely generated over $B_{0}$ since $B$ is noetherian; hence there exists a point $x \in \operatorname{Spec}(A)$ such that $B_{l} \otimes_{A} k(x) \neq 0$. Then the action on the fiber over $x$ is non-trivial. 


\section{LUNA'S FUNDAMENTAL LEMMA FOR DIAGONALIZABLE GROUPS}

Finally, assume that the fibers of $f$ are reduced, and let us show that in this case, condition (v) implies condition (iii). Both conditions are fiberwise, so replacing $f$ and $G$ with the fiber over a point $x$ and the stabilizer $G_{x}$, we can assume that $X=\operatorname{Spec}(k)$ is $G$-invariant and that for any point $y \in Y=\operatorname{Spec}(A)$, one has $G_{y}=G$. Fix $0 \neq n \in L$; then the latter condition implies that $A_{n}$ is contained in all $L$-prime ideals of $A$. By Lemma 4.4 .8 , the module $A_{n}$ lies in the nilpotent radical, and since $A$ is reduced by the assumption, $A=A_{0}$, as claimed.

5.5.3 Inert morphisms. Consider a $G$-equivariant morphism $f: X^{\prime} \rightarrow X$ and assume that it takes special orbits (Section 5.1.8) to special orbits. We say that

(i) the morphism $f$ is pointwise inert if condition ( $\mathrm{v}$ ) of Lemma 5.5.2 holds, namely it is fixedpoint reflecting;

(ii) the morphism $f$ is fiberwise inert if any of the conditions (ii), (iii), and (iv) of Lemma 5.5.2 hold, namely, for each point $x \in X$, the stabilizer $G_{x}$ acts trivially on the fiber $X^{\prime} \times_{X} x$;

(iii) the morphism is inert if condition (i) of Lemma 5.5.2 holds, namely, it is inertia preserving.

Remark 5.5.4. (i) One can easily construct a morphism with non-reduced fibers which is pointwise inert but not fiberwise inert. We have not studied the difference (if any!) between inert morphisms and morphisms which are fiberwise inert.

(ii) It follows directly from the definition (Section 5.3.1) that any strongly equivariant morphism is inert and, conversely, pointwise inertness will play a role in our criteria of strong equivariance.

(iii) If any of the conditions of inertness are violated, then the quotient functor might behave rather badly. For example, the morphism is not inertia preserving in Example 5.2.7(i) and (i)', and it takes special orbits to non-special orbits in Example 5.2.7(ii) and (ii)'.

5.5.5 Comparison with the literature. In the case of a reductive group $G$ and a $G$-equivariant étale morphism of varieties, the fixed-point reflecting condition was used by Luna. Although not formulated separately, it is contained in [Lun73, Lemme fondamental]. For finite groups, fixedpoint reflecting morphisms were called inert in [ILO14, Section VIII.5.3.6]. In both cases, the condition on special orbits was not required since it was automatically satisfied. An analogue of our version of inert morphisms was called "schematically inert" in [ILO14, Remark VIII.5.6.2].

An analogue of inertness and pointwise inertness is used by Alper in a much more general context of good and adequate moduli spaces; see [Alp10, § 2.1,2.2].

\subsection{Main results about the quotient functor}

In this section, we will prove that pointwise inert regular morphisms are strongly regular. In the case of diagonalizable groups, this extends the classical Luna's fundamental lemma, which deals with inert étale morphisms of varieties, to regular morphisms of schemes. Moreover, we will obtain general criteria for equivariant morphisms to be strongly equivariant and establish descent for syntomic and lci morphisms.

5.6.1 Local case. We start with the following extension of Theorem 4.8.1 to the case of local actions that do not have to be strictly local.

Lemma 5.6.2. Assume that locally noetherian schemes $X$ and $X^{\prime}$ are provided with relatively affine actions of a diagonalizable group $G=\mathbf{D}_{L}$ and that $f: X^{\prime} \rightarrow X$ is a $G$-equivariant morphism with quotient $g=f / / G: Y^{\prime} \rightarrow Y$. Let $x^{\prime} \in X^{\prime}$ be a point with images $x=f\left(x^{\prime}\right) \in X, y^{\prime} \in Y^{\prime}$, 


\section{Abramovich and M. Temkin}

and $y \in Y$. Assume that

(a) $x$ lies in a special orbit;

(b) the inclusion of the stabilizers $G_{x^{\prime}} \subseteq G_{x}$ is an equality;

(c) for $G_{x}=\mathbf{D}_{L_{x}}$, the $L_{x}$-grading on the vector spaces $\Omega_{X^{\prime} / X} \otimes k\left(x^{\prime}\right)$ and $H_{1}\left(\mathbb{L}_{X^{\prime} / X} \otimes^{\mathrm{L}} k\left(x^{\prime}\right)\right)$ are trivial.

Then:

(i) The morphism $f$ is strongly equivariant over $y^{\prime}$.

(ii) The morphism $f$ is formally smooth at $x^{\prime}$ if and only if $g$ is formally smooth at $y^{\prime}$.

(iii) If $f$ is lci at $x^{\prime}$, then $g$ is lci at $y^{\prime}$. Conversely, if $g$ is lci at $y^{\prime}$ and the homomorphisms $\mathcal{O}_{Y, y} \rightarrow \mathcal{O}_{Y^{\prime}, y^{\prime}}$ and $\mathcal{O}_{Y, y} \rightarrow \mathcal{O}_{X, x}$ are Tor-independent, then $f$ is lci at $x^{\prime}$. In particular, $f$ is syntomic at $x^{\prime}$ if and only if $g$ is syntomic at $y^{\prime}$.

Proof. The claim is local at $y^{\prime}$ and $y$, so we can assume $Y^{\prime}=\operatorname{Spec} \mathcal{O}_{Y^{\prime}, y^{\prime}}$ and $Y=\operatorname{Spec} \mathcal{O}_{Y, y}$. Then the actions on $X^{\prime}$ and $X$ are local (Section 5.1.9), and $x$ lies in the closed orbit by assumption (a). The inverse implications in parts (ii) and (iii) easily follow from part (i). So we need to prove that $f$ is strongly equivariant and that if $f$ is formally smooth or lci, then $g$ has the same property. Let us recall two cases of the lemma that were already established earlier.

Case 1: The lemma holds true when $G_{x}=G$. Indeed, in this case the closed orbits are $x$ and $x^{\prime}$, so $X=\operatorname{Spec}(A)$ and $X^{\prime}=\operatorname{Spec}\left(A^{\prime}\right)$, where $A$ and $A^{\prime}$ are strictly $L$-local rings by assumption (b). So case 1 is covered by Theorem 4.8.1.

Case 2. The lemma holds true when $G_{x}=\{1\}$. Indeed, in this case the actions are free by Lemma 5.4.4; hence case 2 is covered by Corollary 5.4.8.

Now, assume that $G_{x}$ is arbitrary. Recall that the actions of $G^{\prime}:=G / G_{x}$ on $Z=X / / G_{x}$ and $Z^{\prime}=X^{\prime} / / G_{x}$ are free by Corollary 5.4.5. Consider the $G^{\prime}$-equivariant morphism $h=f / / G_{x}$. By case 1 above, $f$ is strongly equivariant over the image $z^{\prime} \in Z^{\prime}$ of $x^{\prime}$, and if $f$ is formally smooth or lci at $x^{\prime}$, then $h$ is formally smooth or lci at $z^{\prime}$. By the freeness of the action, $Z_{y}$ consists of a single orbit, so $G^{\prime}, h$, and $z^{\prime}$ satisfy all assumptions of the lemma. Therefore case 2 applies, and we obtain that $h$ is strongly equivariant over $y^{\prime}$, and if $f$ is formally smooth or lci at $x^{\prime}$, then $g=h / / G^{\prime}$ is formally smooth or lci at $y^{\prime}$, respectively. The lemma follows.

\subsubsection{Luna's fundamental lemma.}

Theorem 5.6.4. Let $X$ and $X^{\prime}$ be locally noetherian schemes provided with relatively affine actions of a diagonalizable group $G$, and let $f: X^{\prime} \rightarrow X$ be a $G$-equivariant morphism.

(i) The morphism $f$ is strongly equivariant if and only if $f$ is fiberwise inert and any special orbit in $X^{\prime}$ contains a point $x^{\prime}$ such that the action of $G_{x^{\prime}}$ on $H_{1}\left(\mathbb{L}_{X^{\prime} / X} \otimes^{\mathrm{L}} k\left(x^{\prime}\right)\right)$ is trivial.

(ii) Let $P$ be one of the following properties: (a) regular, (b) smooth, (c) étale, (d) an open embedding. Then the following conditions are equivalent: (1) $f$ is pointwise inert and satisfies $P$, (2) $f$ is inert and satisfies $P,(3) f$ is strongly equivariant and satisfies $P$, (4) $f$ strongly satisfies $P$.

(iii) The morphism $f$ is strongly syntomic if and only if it is syntomic and strongly equivariant. Moreover, if $f$ is strongly equivariant, then the following claims hold: (a) if $f$ is lci, then $f / / G$ is lci, (b) if $f / / G$ is lci and Tor-independent with the morphism $X \rightarrow X / / G$, then $f$ is lci.

Part (iii) refers to Avramov's definition of lci morphisms and the resulting notion of syntomic morphisms described in Section 4.7.9. 


\section{LUNA'S FUNDAMENTAL LEMMA FOR DIAGONALIZABLE GROUPS}

Proof. (i) The direct implication is clear, so let us prove the inverse implication. Let $y^{\prime} \in X^{\prime} / / G$ be a point, and choose a point $x^{\prime}$ in the special orbit over $y^{\prime}$ such that the action of $G_{x^{\prime}}$ on $H_{1}\left(\mathbb{L}_{X^{\prime} / X} \otimes^{\mathrm{L}} k\left(x^{\prime}\right)\right)$ is trivial. We assume that $f$ is fiberwise inert, so (a) $x=f\left(x^{\prime}\right)$ lies in a special orbit, (b) $G_{x^{\prime}}=G_{x}$, and (c) by Lemma 5.5.2, the action of $G_{x^{\prime}}$ on $H_{0}\left(\mathbb{L}_{X^{\prime} / X} \otimes^{\mathrm{L}} k\left(x^{\prime}\right)\right)=$ $\Omega_{X^{\prime} / X} \otimes k\left(x^{\prime}\right)$ is trivial as well. Thus we may apply Lemma 5.6.2 at $x^{\prime}$, obtaining that $f$ is strongly equivariant over $y^{\prime}$ and proving part (i). Moreover, this argument shows that if $f$ is regular, then $g=f / / G$ is formally smooth at all point of $X / / G$ and hence is itself a regular morphism.

Part (iii) follows from Lemma 5.6.2 similarly.

(ii) The implications (4) $\Longrightarrow(3) \Longrightarrow(2) \Longrightarrow(1)$ are obvious, so let us prove $(1) \Longrightarrow(4)$. Regardless of the choice of $P$, the morphism $f$ is regular. Since $f$ has reduced fibers, it is fiberwise inert by Lemma 5.5.2, and as we have proved above, it is then strongly regular. This covers property (a). A morphism is smooth if and only if it is regular and of finite type. Hence property (b) follows from property (a) and Corollary 5.2.4. A smooth morphism is étale or an open embedding if and only if it has finite fibers or is a monomorphism, respectively. Hence properties (c) and (d) follow from property (a) and Proposition 5.3.6(a),(b).

\subsection{Groups of multiplicative type}

Assume that $S$ is a scheme and $G_{S}$ is an $S$-group. We say that $G_{S}$ is of multiplicative type if it is of finite type and $G_{S^{\prime}}=G_{S} \times{ }_{S} S^{\prime}$ is isomorphic to a diagonalizable $S^{\prime}$-group $\mathbf{D}_{L} \times S^{\prime}$ for a surjective, flat, finitely presented morphism $h: S^{\prime} \rightarrow S$. For example, this includes the case when $S=\operatorname{Spec}(\mathbb{Z}[1 / N])$ and $G_{S}=\mathbb{Z} / N \mathbb{Z}$. Our definition is more restrictive than that in [SGA3-2, IX.1.1] because we use the fppf topology instead of the quasi-compact, faithfully flat (fpqc) topology and consider only groups of finite type. Recall that by [SGA3-2, X.4.5], any such group $G_{S}$ is quasi-isotrivial; that is, it can be split by an étale surjective morphism $h: S^{\prime} \rightarrow S$.

We claim that all results of Section 5 extend to the case when an $S$-scheme $X$ is provided with a relatively affine action of an $S$-group $G_{S}$ of multiplicative type. The only exception is part (ii) of Lemma 5.1.10, which makes no sense for non-diagonalizable group. The proofs we provided of the equivalence of parts (i) and (iii) in Lemma 5.1.10 and parts (a), (b) and (c) of Theorem 5.2.2(i) apply to groups of multiplicative type without change. All remaining results, including Luna's fundamental lemma, can be extended to $G_{S}$ of multiplicative type with $L$ denoting the abelian group Cartier dual to $G_{S^{\prime}}$ by use of étale descent with respect to $h$. Namely, $X_{S^{\prime}} / / G_{S^{\prime}}=\left(X / / G_{S}\right) \times_{S} S^{\prime}$, so the result for $X_{S^{\prime}} / / G_{S^{\prime}}$ extends to $X / / G$ by descent. In fact, already flat descent suffices in almost all cases, with the main exception being Proposition 5.1.16.

\section{REFERENCES}

AFH94 L. L. Avramov, H. B. Foxby, and B. Herzog, Structure of local homomorphisms, J. Algebra 164 (1994), no. 1, 124-145; https://doi.org/10.1006/jabr.1994.1057.

AKMW02 D. Abramovich, K. Karu, K. Matsuki, and J. Włodarczyk, Torification and factorization of birational maps, J. Amer. Math. Soc. 15 (2002), no. 3, 531-572; https://doi.org/10.1090/ S0894-0347-02-00396-X.

Alp08 J. Alper, Good moduli spaces for Artin stacks, Ph.D. thesis, Stanford University, 2008, available at http://gradworks. proquest. com/33/13/3313806.html. 


\section{Abramovich and M. Temkin}

Alp10

And74

AOV08

AT16

AT17

Avr99

BR85

EGA

Fog73

FR93

GY83

Har77

Ill71

ILO14

Knu71

Lun73

Mat89

MFK94

SGA3-1

SGA3-2

On the local quotient structure of Artin stacks, J. Pure Appl. Algebra 214 (2010), no. 9, 1576-1591; https://doi.org/10.1016/j.jpaa.2009.11.016.

M. André, Localisation de la lissité formelle, Manuscripta Math. 13 (1974), 297-307; https: //doi.org/10.1007/BF01168230.

D. Abramovich, M. Olsson, and A. Vistoli, Tame stacks in positive characteristic, Ann. Inst. Fourier (Grenoble) 58 (2008), no. 4, 1057-1091; https://doi.org/10.5802/aif.2378.

D. Abramovich and M. Temkin, Functorial factorization of birational maps for qe schemes in characteristic 0, 2016, arXiv:1606.08414.

, Torification of diagonalizable group actions on toroidal schemes, J. Algebra 472 (2017), 279-338; https://doi.org/10.1016/j.jalgebra.2016.10.016.

L.L. Avramov, Locally complete intersection homomorphisms and a conjecture of Quillen on the vanishing of cotangent homology, Ann. of Math. 150 (1999), no. 2, 455-487; https: //doi.org/10.2307/121087.

P. Bardsley and R. W. Richardson, Étale slices for algebraic transformation groups in characteristic p, Proc. London Math. Soc. 51 (1985), no. 2, 295-317; https://doi.org/10.1112/

$\mathrm{plms} / \mathrm{s} 3-51.2 .295$.

A. Grothendieck and J. Dieudonné, Éléments de géométrie algébrique, Publ. Math. Inst. Hautes Études Sci. 4 (Chapter 0, 1-7, and I, 1-10), 8 (II, 1-8), 11 (Chapter 0, 8-13, and III, 1-5), 17 (III, 6-7), 20 (Chapter 0, 14-23, and IV, 1), 24 (IV, 2-7), 28 (IV, 8-15), and 32 (IV, 16-21), 1960-1967; available at http://www. numdam.org.

J. Fogarty, Fixed point schemes, Amer. J. Math. 95 (1973), 35-51; https://doi.org/10. $2307 / 2373642$.

L. Franco and A. G. Rodicio, On the vanishing of the second André-Quillen homology of a local homomorphism, J. Algebra 155 (1993), no. 1, 137-141; https://doi.org/10.1006/ jabr.1993.1035.

S. Goto and K. Yamagishi, Finite generation of Noetherian graded rings, Proc. Amer. Math. Soc. 89 (1983), no. 1, 41-44; https://doi.org/10.2307/2045060.

R. Hartshorne, Algebraic geometry, Grad. Texts in Math., vol. 52 (Springer-Verlag, New YorkHeidelberg, 1977); https://doi.org/10.1007/978-1-4757-3849-0.

L. Illusie, Complexe cotangent et déformations. I, Lecture Notes in Math., vol. 239 (SpringerVerlag, Berlin - New York, 1971); https://doi.org/10.1007/BFb0059052.

L. Illusie, Y. Laszlo, and F. Orgogozo (eds), Travaux de Gabber sur l'uniformisation locale et la cohomologie étale des schémas quasi-excellents (Séminaire à l'École polytechnique 2006-2008), Astérisque (2014), no. 363-364.

D. Knutson, Algebraic spaces, Lecture Notes in Math., vol. 203 (Springer-Verlag, Berlin - New York, 1971); https://doi.org/10.1007/BFb0059750.

D. Luna, Slices étales, in Sur les groupes algébriques, Bull. Soc. Math. France, Paris, vol. 33 (Soc. Math. France, Paris, 1973), 81-105.

H. Matsumura, Commutative ring theory, 2nd ed., Cambridge Stud. Adv. Math., vol. 8 (Cambridge Univ. Press, Cambridge, 1989).

D. Mumford, J. Fogarty, and F. Kirwan, Geometric invariant theory, 3rd ed., Ergeb. Math. Grenzgeb., vol. 34 (Springer-Verlag, Berlin, 1994); https://doi.org/10.1007/ 978-3-642-57916-5.

M. Demazure and A. Grothendieck (eds), Séminaire de Géométrie algébrique du BoisMarie, 1962-1964 (SGA3), Schémas en groupes, Tome 1: Propriétés générales des schémas en groupes, Lecture Notes in Math., vol. 151 (Springer-Verlag, Berlin - New York, 1970); https://doi.org/10.1007/BFb0058993.

M. Demazure and A. Grothendieck (eds), Séminaire de Géométrie algébrique du Bois-Marie, 1962-1964 (SGA3), Schémas en groupes, Tome 2: Groupes de type multiplicatif, et structure 


\section{LUNA'S FUNDAMENTAL LEMMA FOR DIAGONALIZABLE GROUPS}

des schémas en groupes généraux, Lecture Notes in Math., vol. 152 (Springer-Verlag, Berlin New York, 1970); https://doi.org/10.1007/BFb0059005.

SP17 The Stacks Project Authors, Stacks Project, version 2017, available at http://stacks.math. columbia.edu.

Tem04 M. Temkin, On local properties of non-Archimedean analytic spaces. II, Israel J. Math. 140 (2004), no. 1, 1-27; https://doi.org/10.1007/BF02786625.

Tha96 M. Thaddeus, Geometric invariant theory and flips, J. Amer. Math. Soc. 9 (1996), no. 3, 691-723; https://doi.org/10.1090/S0894-0347-96-00204-4.

Wło00 J. Włodarczyk, Birational cobordisms and factorization of birational maps, J. Algebraic Geom. 9 (2000), no. 3, 425-449.

Wło03_ Toroidal varieties and the weak factorization theorem, Invent. Math. 154 (2003), no. 2, 223-331; https://doi.org/10.1007/s00222-003-0305-8.

Dan Abramovich abrmovic@math.brown.edu

Department of Mathematics, Box 1917, Brown University, Providence, RI, 02912, USA

Michael Temkin temkin@math.huji.ac.il

Einstein Institute of Mathematics, The Hebrew University of Jerusalem, Giv'at Ram, Jerusalem, 91904, Israel 